\title{
The structure of piecewise monotonic transformations
}

\author{
FRANZ HOFBAUER † \\ From the Institut für Mathematik, Universität Wien, Austria
}

(Received 3 July 1980 and revised 2 January 1981)

\begin{abstract}
Transformations on $[0,1]$ which are piecewise monotonic and piecewise continuous are considered. Using symbolic dynamics, the structure of their nonwandering set is determined. This is then used to prove results about maximal and absolutely continuous invariant measures.
\end{abstract}

\section{Introduction}

We consider dynamical systems $([0,1], f)$, where $[0,1]=\bigcup_{i=1}^{n} J_{i}$, the $J_{i}$ are disjoint intervals and $f \mid J_{i}$ is continuous and increasing. The $f$-expansion gives rise to a shift space $\Sigma_{f}^{+}$(cf. $\S 1$ ). Our goal is to determine the structure of the non-wandering set $\Omega$ of $\Sigma_{f}^{+}$. In [4] it is shown how these results can be extended to the case where $f \mid J_{i}$ is either increasing or decreasing.

$\S 1$ gives a summary of results proved in [3] and needed in this paper. In $\$ 2$ it is shown that the non-wandering set $\Omega$ of $\Sigma_{f}^{+}$can be written as $\bigcup_{i \geq 1} \Omega_{i} \cup Y \cup Z$. There are finitely or countably many $\Omega_{i}$. The $\Omega_{i}$ and $Y$ are closed, $\sigma$-invariant subsets of $\Sigma_{f}^{+}$, and $\Omega_{i}$ is topologically transitive. $\Omega_{i} \cap \Omega_{j}$, for $i \neq j$, and $\Omega_{i} \cap Y$ are empty or finite; $Z$ is finite and wandering in $\Omega$. The topological entropy of $Y$ is zero. $\Omega_{i}$ is a finite union of intervals, a Cantor set or a periodic orbit. Furthermore, $\Omega_{i}=X_{1} \cup X_{2} \cup \cdots \cup X_{q}(q \geq 1)$, the $X_{i}$ are closed and again pairwise disjoint up to finitely many points; $\sigma\left(X_{i}\right) \subset X_{i+1}$ for $1 \leq i \leq q-1, \sigma\left(X_{q}\right) \subset X_{1}$ and $\sigma^{q} / X_{i}$ is topologically mixing.

The rest of the paper deals with invariant measures. It is shown in [3] that $\boldsymbol{\Omega}_{i}$ has a unique measure with maximal entropy if $h_{\text {top }}\left(\Omega_{i}\right)>0$. In $\S 3$ this measure is characterized as the measure with respect to which the periodic points are uniformly distributed. $\S 4$ considers invariant measures of $([0,1], f)$ which are absolutely continuous with respect to Lebesgue measure and gives an example in connection with this.

Finally, I should like to thank Z. Nitecki for pointing out an error which was contained in the first version of this paper. 


\section{Preliminaries}

We give a description of results proved in [3]. Let $f$ be as in the introduction. Define the $f$-expansion $\phi:[0,1] \rightarrow \Sigma_{n}^{+}=\{1, \ldots, n\}^{N}$ by $\phi(x)=i_{0} i_{1} i_{2} \ldots$, where $i_{j}$ is the number $i$ of the interval $J_{i}$ with $f^{j}(x) \in J_{i}$. If $J_{k}=(r, s)$, define $\mathbf{a}^{k}=\lim _{t \downarrow r} \phi(t)$ and $\mathbf{b}^{k}=\lim _{i \uparrow s} \phi(t)$. Set

$$
\Sigma_{f}^{+}=\left\{\mathbf{x}=x_{0} x_{1} \cdots \in \Sigma_{n}^{+}: \mathbf{a}^{x_{m}} \leq x_{m} x_{m+1} \cdots=\sigma^{m} \mathbf{x} \leq \mathbf{b}^{x_{m}} \forall m \geq 0\right\},
$$

where $\leq$ denotes the lexicographic ordering and $\sigma$ the shift transformation. We have $\overline{\phi([0,1])}=\Sigma_{f}^{+}, \Sigma_{f}^{+} \backslash \phi([0,1])$ is countable, $\phi$ is order preserving and $\sigma \circ \phi=$ $\phi \circ f . \phi$ is injective if and only if $\left(J_{1}, J_{2}, \ldots, J_{n}\right)$ is a generator for $([0,1], f)$.

$\Sigma_{f}^{+}$can be characterized by all blocks $x_{0} x_{1} \ldots x_{m-1}$ which are admissible in $\Sigma_{f}^{+}$, i.e. $0\left[x_{0} x_{1} \ldots x_{m-1}\right]=\left\{\mathbf{z} \in \Sigma_{f}^{+}: z_{i}=x_{i}\right.$ for $\left.0 \leq i \leq m-1\right\}$ is not empty. This is equivalent to $\sigma^{m-1}\left({ }_{0}\left[x_{0} \ldots x_{m-1}\right]\right) \neq \varnothing$. We have ${ }_{0}\left[x_{0}\right]=\left[\mathbf{a}^{x_{0}}, \mathbf{b}^{x_{0}}\right]$, which denotes a closed interval in $\Sigma_{f}^{+}$. We show by induction that there are $i, j, k \geq 1$ and $l \geq 1$ such that

$$
\sigma^{m-1}\left({ }_{0}\left[x_{0} \cdots x_{m-1}\right]\right)=\left[\sigma^{k-1} \mathbf{a}^{i}, \sigma^{l-1} \mathbf{b}^{j}\right] \text { with } a_{k-r}^{i}=b_{l-r}^{j} \quad \text { for } 1 \leq r \leq \min (k, l) .
$$

The induction step is

$$
\begin{aligned}
\sigma^{m}\left({ }_{0}\left[x_{0} \cdots x_{m}\right]\right) & ={ }_{0}\left[x_{m}\right] \cap \sigma^{m}\left({ }_{0}\left[x_{0} \cdots x_{m-1}\right]\right) \\
& =\left[\mathbf{a}^{x_{m}}, \mathbf{b}^{x_{m}}\right] \cap\left[\sigma^{k} \mathbf{a}^{i}, \sigma^{l} \mathbf{b}^{i}\right] \\
& =\left\{\begin{array}{lll}
\varnothing, & \text { if } x_{m}<a_{k}^{i} \text { or } x_{m}>b_{l}^{i}, \\
{\left[\sigma^{k} \mathbf{a}^{i}, \mathbf{b}^{x_{m}}\right],} & \text { if } x_{m}=a_{k}^{i} \text { and } x_{m}<b_{l}^{i} \\
{\left[\mathbf{a}^{x_{m}}, \sigma^{l} \mathbf{b}^{j}\right],} & \text { if } x_{m}>a_{k}^{i} \text { and } x_{m}=b_{l}^{j} \\
{\left[\sigma^{k} \mathbf{a}^{i}, \sigma^{l} \mathbf{b}^{j}\right],} & \text { if } x_{m}=a_{k}^{i}=b_{l}^{i} \\
{\left[\mathbf{a}^{x_{m}}, \mathbf{b}^{x_{m}}\right],} & \text { if } a_{k}^{i}<x_{m}<b_{l}^{j}
\end{array}\right.
\end{aligned}
$$

It is easy to see that $\left.\sigma^{m}{ }_{0}\left[x_{0} \cdots x_{m}\right]\right)$ is either empty or satisfies (1.2). Hence we have shown (1.2) by induction. In particular, $\sigma^{m-1}\left({ }_{0}\left[x_{0} \cdots x_{m-1}\right]\right)$ is a closed sub-interval of some ${ }_{0}[i] \subset \Sigma_{f}^{+}(1 \leq i \leq n)$. If it is not empty, it is either $\sigma^{k-1}\left({ }_{0}\left[a_{0}^{i} \cdots a_{k-1}^{i}\right]\right)$ or $\sigma^{k-1}\left({ }_{0}\left[b_{0}^{i} \cdots b_{k-1}^{i}\right]\right)$ for some $i$ and $k$, because all intervals one obtains by repeated use of $(1.3)$ are such sets:

$$
\left[\sigma^{k-1} \mathbf{a}^{i}, \sigma^{l-1} \mathbf{b}^{j}\right]=\sigma^{k-1}\left(0\left[a_{0}^{i} \cdots a_{k-1}^{i}\right]\right) \quad \text { if } k \geq l
$$

and

$$
\left[\sigma^{k-1} \mathbf{a}^{i}, \sigma^{l-1} \mathbf{b}^{i}\right]=\sigma^{l-1}\left({ }_{0}\left[b_{0}^{j} \cdots b_{l-1}^{i}\right]\right) \quad \text { if } k \leq l .
$$

This follows from lemma 11 of [3]. Hence many of the sets $\sigma^{m-1}\left({ }_{0}\left[x_{0} \cdots x_{m-1}\right]\right)$ coincide.

We form a diagram with the sets $\sigma^{m-1}\left({ }_{0}\left[x_{0} \cdots x_{m-1}\right]\right.$ ) (we take $n=2$ for convenience). It will be called $M$. 


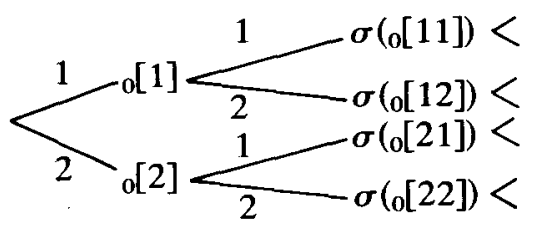

There is a 1-1 correspondence between paths in $M$ which begin with one of the arrows ending at some ${ }_{0}[k](1 \leq k \leq n)$ and which do not lead to an empty set and the points $\mathrm{x} \in \Sigma_{f}^{+}\left(x_{0}, x_{1} \cdots\right.$ are the numbers of the arrows on this path). We shall call such paths special paths in order to distinguish them from paths beginning with any other arrow of (1.4) (they represent also points of $\Sigma_{f}^{+}$, but an $\mathbf{x} \in \Sigma_{f}^{+}$may have many such representations).

$M$ serves also for another purpose. Set

$$
\begin{aligned}
D & =\left\{\sigma^{m-1}\left({ }_{0}\left[x_{0} \cdots x_{m-1}\right]\right): \sigma^{m-1}\left({ }_{0}\left[x_{0} \cdots x_{m-1}\right]\right) \neq \varnothing\right\} \\
& =\left\{\sigma^{m-1}\left({ }_{0}\left[a_{0}^{i} \cdots a_{m-1}^{i}\right]\right), \sigma^{m-1}\left({ }_{0}\left[b_{0}^{i} \cdots b_{m-1}^{i}\right]\right): 1 \leq i \leq n, m \geq 1\right\} .
\end{aligned}
$$

Together with the arrows $\sigma^{m-1}\left({ }_{0}\left[x_{0} \cdots x_{m-1}\right]\right) \stackrel{x_{m}}{\longrightarrow} \sigma^{m}\left({ }_{0}\left[x_{0} \cdots x_{m}\right]\right), D$ becomes the diagram $M$ of (1.4). In [3] we have used $\sigma^{m}\left({ }_{0}\left[x_{0} \cdots x_{m-1}\right]\right)$ instead of $\sigma^{m-1}\left({ }_{0}\left[x_{0} \cdots x_{m-1}\right]\right)$. This makes no difference for the results and the proofs of [3], but the new definition is more convenient. For example, $D$ need not be a set of pairs $\left(x_{m-1}, \sigma^{m}\left(0\left[x_{0} \cdots x_{m-1}\right]\right)\right)$ as in [3] because $x_{m-1}$ is determined by $\sigma^{m-1}\left({ }_{0}\left[x_{0} \cdots x_{m-1}\right]\right) \subset_{0}\left[x_{m-1}\right]$.

Define $\Sigma_{M}=\left\{\mathbf{y} \in D^{\mathbf{Z}}\right.$ : there is an arrow from $y_{i}$ to $y_{i+1}$ in $\left.M \forall i \in \mathbb{Z}\right\}$. Now $\Sigma_{f}=$ $\left\{\mathbf{x} \in\{1, \ldots, n\}^{\mathbf{Z}}: x_{m} x_{m+1} \cdots \in \Sigma_{f}^{+} \forall m \in \mathbb{Z}\right\}$, the natural extension of $\Sigma_{f}^{+}$, can be written as disjoint union of sets $N$ and $X$ which are $\sigma$-invariant and measurable. $N$ contains no periodic points and is a null set for every measure with maximal entropy. $(X, \sigma)$ and $\left(\Sigma_{M}, \sigma\right)$ are isomorphic, the isomorphism $\psi$ is given by representing $\mathbf{y} \in \Sigma_{M}$, which is a two-sided path of vertices in the diagram $M$, by the numbers of the arrows on this path giving an $x \in X \subset \Sigma_{f}$ (cf. [3]). Two-sided paths exist in $M$ because many of the sets $\sigma^{m-1}\left({ }_{0}\left[x_{0} \cdots x_{m-1}\right]\right)$ coincide. Examples can be found in [3]. The map $\chi:\left(\Sigma_{M}, \sigma\right) \stackrel{\psi}{\rightarrow}\left(\Sigma_{f}, \sigma\right) \stackrel{\pi}{\rightarrow}\left(\Sigma_{f}^{+}, \sigma\right)$ is the composition of this isomorphism $\psi$ and the projection $\pi$ to positive coordinates. $\chi$ is continuous.

We conclude $\S 1$ with two remarks. This first one explains how the results of this paper can be extended to maps $f$, for which $f \mid J_{i}$ is continuous and increasing for some is and decreasing for the other is. The only difference to the piecewise increasing case is that we have another order relation in the shift space such that $\phi$ is order preserving (cf. [4]). $\Sigma_{f}^{+}$is defined as in (1.1), but with this different order relation. $\sigma$ is then not order preserving, hence the intervals occurring in (1.3) may have also $\sigma^{k} \mathbf{b}^{i}$ for some $i$ and $k$ as initial point or $\sigma^{l} \mathbf{a}^{j}$ for some $j$ and $l$ as endpoint or both. One can define the diagram $M$ as in (1.4) and also the map $\chi$. In [4], a piecewise increasing transformation $g$ is constructed such that $\left(\Sigma_{f}^{+}, \sigma\right)$ is a two-toone factor of $\left(\Sigma_{g}^{+}, \sigma\right)$. The only proofs in this paper which will use the explicit form of the intervals in (1.3) are those of lemmas 1,4 and (ii) of lemma 7 . These proofs 
can be extended to the piecewise increasing-decreasing case in the same way as one obtains the diagram $M$ for $f$ from that of $g$ (cf. [4]). The definitions and all other proofs work unchanged. Hence all results of this paper are also valid for piecewise increasing-decreasing transformations.

The second remark shows how one can determine the structure of the nonwandering set of $([0,1], f)$ from that of $\left(\Sigma_{f}^{+}, \sigma\right)$. If $\left(J_{1}, \ldots, J_{n}\right)$ is a generator for $([0,1], f)$, then $\phi$ is injective and $\phi^{-1}$ can be easily extended on all of $\Sigma_{f}^{+}$to a map $\rho$ which is continuous and preserves the ordering. An $x \in[0,1]$ is wandering under $f$ if and only if $\phi(x) \notin \Omega$, unless $x$ is an inverse image under some iterate of $f$ of an endpoint of some $J_{i}$, not equal to 0 or 1 . which can be non-wandering, and $\phi(x) \notin \Omega$. These are exactly those $x \in[0,1]$ such that $\rho^{-1}(x)$ is not a single point but two points $\mathbf{x}$ and $\mathbf{x}^{\prime}$. If $x$ is non-wandering and $\mathbf{x} \notin \Omega, x^{\prime} \notin \Omega$, then there is an $\varepsilon>0$ such that $f^{k}(x-\varepsilon, x) \cap(x-\varepsilon)=\varnothing$ and $f^{k}(x, x+\varepsilon) \cap(x, x+\varepsilon)=\varnothing$ for all $k \geq 1$ (the intervals $(x-\varepsilon, x)$ and $(x, x+\varepsilon)$ correspond to neighbourhoods of $\mathbf{x}$ and $\mathbf{x}^{\prime}$ respectively). Hence $x$ is isolated in the non-wandering set of $([0,1], f)$ and non-periodic (otherwise $\mathbf{x}$ or $\mathbf{x}^{\prime}$ is periodic). If we transfer the structure of $\Omega$ to $([0,1], f)$ via $\rho$, then we can add these points to $\rho(Z)$. Hence the non-wandering set of $([0,1], f)$ has the same structure as that of $\left(\Sigma_{f}^{+}, \sigma\right)$ described in $\S 0$ : one has only to allow that the set corresponding to $Z$ is countable.

If $\left(J_{1}, \ldots, J_{n}\right)$ is not a generator, then $\phi$ maps certain intervals to single points (cf. [4]).

2. The non-wandering set of $\left(\Sigma_{f}^{+}, \sigma\right)$

We show that $\left(\Sigma_{f}^{+}, \sigma\right)$ has the structure described in $\S 0$. To this end we consider $M$ as a $0-1$-matrix with index set $D . M_{d e}=1$ if and only if there is an arrow from $d$ to $e$ in $M$. We divide $M$ into irreducible submatrices $M_{i}$ with index set $D_{i}(i \geq 1)$, i.e. $D_{i}$ is a maximal subset of $D$ such that, if $d, e \in D_{i}$, then there is a path from $d$ to $e$ in $M$, and $M_{i}=M / D_{i} . \Sigma_{M_{i}} \subset \Sigma_{M}$ denotes the shift space corresponding to $M_{i}$. If $i \neq j$, then $D_{i} \cap D_{i}=\varnothing$ and $\bigcup_{i \geq 1} D_{i} \subset D$. It may happen that $\bigcup_{i \geq 1} D_{i} \neq D$. As

$$
D=\left\{\sigma^{m}\left({ }_{0}\left[a_{0}^{i} \cdots a_{m}^{i}\right]\right), \sigma^{m}\left({ }_{0}\left[b_{0}^{i} \cdots b_{m}^{i}\right]\right): 1 \leq i \leq n, m \geq 0\right\}
$$

and because of the arrows

$$
\begin{aligned}
& \sigma^{m}\left({ }_{0}\left[a_{0}^{i} \cdots a_{m}^{i}\right]\right) \rightarrow \sigma^{m+1}\left({ }_{0}\left[a_{0}^{i} \cdots a_{m+1}^{i}\right]\right), \\
& \sigma^{m}\left(\left[b_{0}^{i} \cdots b_{m}^{i}\right]\right) \rightarrow \sigma^{m+1}\left({ }_{0}\left[b_{0}^{i} \cdots b_{m+1}^{i}\right]\right),
\end{aligned}
$$

it is easy to see that, for every $D_{i}$, there are $p_{i}, q_{j}, u_{i}, v_{j}(1 \leq j \leq n)$ with $0 \leq p_{j} \leq q_{j} \leq \infty$ and $0 \leq u_{i} \leq v_{i} \leq \infty$ such that

$$
D_{i}=\left\{\sigma^{l}\left({ }_{0}\left[a_{0}^{i} \cdots a_{l}^{i}\right]\right), \sigma^{m}\left({ }_{0}\left[b_{0}^{k} \cdots b_{m}^{k}\right]\right): 1 \leq j, k \leq n, \quad p_{i} \leq l<q_{i}, u_{k} \leq m<v_{k}\right\} .
$$

We introduce an order relation among the $D_{s}$ as follows:

$$
D_{i} \leq D_{j} \text {, if there is a path from } D_{i} \text { to } D_{j} \text { in } M \text {. }
$$


As the $M_{i} \mathrm{~s}$ are the irreducible submatrices of $M$, this is an order relation. Let the indices $i \in \mathbb{N}$ of the $D_{i}$ be such that $D_{i} \leq D_{i}$ implies $i \leqslant j$. Set

$$
\begin{aligned}
& \bar{D}_{i}=\left\{d \in D: \text { there is a path from some } e \in \bigcup_{j \geq i} D_{j} \text { to } d\right\} \\
& D_{i}^{\prime}=\bar{D}_{i} \backslash D_{i} .
\end{aligned}
$$

We then have $\bar{D}_{i} \supset D_{i}^{\prime} \supset \bar{D}_{i+1}$. It is easy to see that $\bar{D}_{i}$ and $D_{i}^{\prime}$ are subsets $C$ of $D$, which have the following property.

If $d \in C$ and there is a path from $d$ to $e$ in $M$, then $e \in C$.

Remark. Suppose $\sigma^{k}\left({ }_{0}\left[a_{0}^{i} \cdots a_{k}^{i}\right]\right) \in D$ (or $\sigma^{k}\left({ }_{0}\left[b_{0}^{i} \cdots b_{k}^{i}\right]\right)$ ) consists only of the single point $y=\sigma^{k} \mathbf{a}^{i}\left(\sigma^{k} \mathbf{b}^{i}\right)$ such that $\sigma^{m} \mathbf{y}$ is not periodic for $m \geq k$. Then $\sigma^{m}\left({ }_{0}\left[a_{0}^{i} \cdots a_{m}^{i}\right]\right)=\left\{\sigma^{m} a^{i}\right\} \in D$ has only one successor in $M$ for all $m \geq k$. We then cancel the vertices $\sigma^{m}\left({ }_{0}\left[a_{0}^{i} \cdots a_{m}^{i}\right]\right)$ for $m \geq k$ in $M$ and the corresponding (via $\left.\chi^{-1}\right)$ set $\bigcup_{j=0}^{\infty} \sigma^{-i}\left(\sigma^{k} a^{i}\right)$ in $\Sigma_{f}^{+}$which is open, countable, $\sigma^{-1}$-invariant and consists only of wandering points. We denote the remainder of $\Sigma_{f}^{+}$again by $\Sigma_{f}^{+}$, which is closed, $\sigma$-invariant and contains all non-wandering points of the original $\Sigma_{f}^{+}$. After this modification, every element of $D$ is either a non-trivial interval or a single point $y$, such that $\sigma^{k} \mathbf{y}$ is periodic for some $k$. As there are only finitely many $\mathbf{a}^{i}$ and $\mathbf{b}^{i}$, there are among the elements of $D$ only finitely many single points, i.e. trivial intervals.

LEMMA 1. Let $C \subset D$ have the property (2.3). Then $\bigcup\{d: d \in C\}$ is a finite union of intervals and is $\sigma$-invariant.

Proof. Set $\mathfrak{U}_{j}=\left\{d \in C: d=\left[\mathbf{a}^{j}, \sigma^{l} \mathbf{b}^{k}\right]\right.$ for some $\left.l, k\right\}$ and $\mathfrak{B}_{j}=\left\{d \in C: d=\left[\sigma^{l} \mathbf{a}^{k}, \mathbf{b}^{j}\right]\right.$ for some $l, k\} . A_{j}=\bigcup_{d \in \mathfrak{Q}_{j}} d$ and $B_{j}=\bigcup_{d \in \mathfrak{Y}_{j}} d$ are intervals in $\Sigma_{f}^{+}$or are empty. Let $d \in D$ be non-trivial and $\gamma_{d}$ the minimal number of steps to go on a path in $M$ from $d \in \mathfrak{A}_{i}$ or $\mathfrak{B}_{j}$ to an element of some $\mathfrak{U}_{m}$ or $\mathfrak{B}_{m}$. Because $d$ is not a single point, $\gamma_{d}<\infty$. This part of $M$ looks as follows $\left(d=\left[\mathbf{a}^{j}, \sigma^{l} \mathbf{b}^{k}\right]\right.$, cf. (1.3)):

$$
\begin{aligned}
& {\left[\mathbf{a}^{j}, \sigma^{l} \mathbf{b}^{k}\right] \rightarrow \cdots \rightarrow } {\left[\begin{array}{c}
\left.\sigma^{\gamma_{d}-1} \mathbf{a}^{j}, \sigma^{l+\gamma_{d}-1} \mathbf{b}^{k}\right] \\
\downarrow \\
\downarrow
\end{array}\right.} \\
& {\left[\sigma^{\gamma_{d}} \mathbf{a}^{j}, \mathbf{b}^{s}\right]\left[\mathbf{a}^{s+1}, \mathbf{b}^{s+1}\right] \cdots\left[\mathbf{a}^{t-1}, \mathbf{b}^{t-1}\right] . }
\end{aligned}
$$

Set $\alpha_{i}=\min \left\{\gamma_{d}: d \in \mathfrak{A}_{j}\right\}$ and $\beta_{j}=\min \left\{\gamma_{d}: d \in \mathfrak{B}_{j}\right\}$. We show that

$$
\sigma^{\alpha_{i}}\left(A_{j}\right) \subset B_{s} \cup A_{t} \cup \bigcup_{s<k<t} A_{k} \quad \text { for some } s \text { and } t(s<t) .
$$

Let $d=\left[\mathbf{a}^{j}, \sigma^{l} \mathbf{b}^{k}\right] \in \mathfrak{U}_{j}$ with $\gamma_{d}=\alpha_{j}$. Then

$$
\begin{gathered}
\sigma^{\alpha_{i}}(d)=\left[\sigma^{\alpha_{i}} \mathbf{a}^{j}, \mathbf{b}^{s}\right] \cup\left[\mathbf{a}^{s+1}, \mathbf{b}^{s+1}\right] \cup \cdots \cup\left[\mathbf{a}^{t-1}, \mathbf{b}^{t-1}\right] \\
\cup\left[\mathbf{a}^{t}, \sigma^{l+\alpha_{i}} \mathbf{b}^{k}\right] \subset B_{s} \cup A_{t} \cup \bigcup_{s<k<t} A_{k} .
\end{gathered}
$$


If $\gamma_{d}>\alpha_{j}$, then

$$
\sigma^{\alpha_{i}}(d)=\left[\sigma^{\alpha_{j}} \mathbf{a}^{j}, \sigma^{l+\alpha_{i} \mathbf{b}^{k}}\right], \sigma^{l+\alpha_{i j}} \mathbf{b}^{k} \leq \mathbf{b}^{s},
$$

because $b_{l+\alpha_{j}}^{k}=a_{\alpha_{j}}^{j}=s$ (cf. (1.2)) and $\mathbf{b}^{k} \in \Sigma_{f}^{+}$(cf. (1.1)). Hence

$$
\sigma^{\alpha_{j}}(d) \subset\left[\sigma^{\alpha_{i}} \mathbf{a}^{j}, \mathbf{b}^{s}\right] \subset B_{s} .
$$

Similar results hold for $d \in \mathfrak{B}_{j}$. Hence

$$
\bigcup_{d \in C} d=\bigcup_{j=1}^{n}\left(\bigcup_{k=0}^{\alpha_{i}-1} \sigma^{k}\left(A_{j}\right) \cup \bigcup_{k=0}^{\beta_{i}-1} \sigma^{k}\left(B_{i}\right)\right) \cup \bigcup_{d \in T} d,
$$

where $T \subset C$ is a finite (or empty) set whose elements are trivial intervals (cf. the remark above). This is a finite union of intervals in $\Sigma_{f}^{+}$. It is $\sigma$-invariant, because $C$ satisfies (2.3).

By lemma 1, $F_{i}:=\bigcup\{d: d \in \bar{D}\}$ and $G_{i}:=\bigcup\left\{d: d \in D_{i}^{\prime}\right\}$ are finite unions of intervals which are $\sigma$-invariant. We have $F_{i} \supset G_{i} \supset F_{i+1}$. We set $G_{0}:=\Sigma_{f}^{+}$. Hence we have split $\Sigma_{f}^{+}$into a sequence of decreasing sets which are $\sigma$-invariant. For $i \geq 1$ we define

$$
\Omega_{i}=\bigcap_{k=0}^{\infty} \overline{\sigma^{-k}\left(F_{i} \backslash G_{i}\right)},
$$

and for $i \geq 0$ we define

$$
\tilde{\Omega}_{i}=\bigcap_{k=0}^{\infty} \overline{\sigma^{-k}\left(G_{i} \backslash F_{i+1}\right)} .
$$

If $D_{i+1}$ does not exist, we set $F_{i+1}=\varnothing$. $\tilde{\Omega}_{i}$ may be empty. As $\Omega_{i} \subset \overline{F_{i} \backslash G_{i}}$ and $\tilde{\Omega}_{i} \subset \bar{G}_{i} \backslash F_{i+1}$, which are finite unions of closed intervals, it follows that the sets $\Omega_{i} \cap \Omega_{j}, \tilde{\Omega}_{i} \cap \tilde{\Omega}_{j}$, for $i \neq j$, and $\Omega_{i} \cap \tilde{\Omega}_{j}$, for any $i$ and $j$, are at most finite.

If there are infinitely many $M_{i}$, we have to consider also an $\tilde{\Omega}_{\infty}$ and an $\Omega_{\infty}$. Set $D_{\infty}^{\prime}=\bigcap_{i=1}^{\infty} \bar{D}_{i}$. This set has the property (2.3), because every $\bar{D}_{i}$ has the property (2.3). Hence $G_{\infty}:=\bigcup\left\{d: d \in D_{\infty}^{\prime}\right\}$ and $\tilde{\Omega}_{\infty}:=\tilde{G}_{\infty}$ are finite unions of intervals which are $\sigma$-invariant. Because $\tilde{\Omega}_{\infty} \subset \bar{F}_{i+1}$ for all $i$, we have that $\Omega_{i} \cap \tilde{\Omega}_{\infty}$ and $\tilde{\Omega}_{i} \cap \tilde{\Omega}_{\infty}$ are at most finite. If there are only finitely many $M_{i}$ s, we set $\tilde{\Omega}_{\infty}=\varnothing$. Now set $H_{i}=\overline{F_{i} \backslash G_{\infty}}$. Then $H_{i}$ is closed and $H_{i} \supset H_{i+1}$. Hence $H=\bigcap_{i \geq 1} H_{i}$ is closed and not empty. Set $\Omega_{\infty}=\bigcap_{k=0}^{\infty} \sigma^{-k}(H)$, which is closed and $\sigma$-invariant. If $D_{\infty}^{\prime}=\varnothing$, then $\Omega_{\infty}=H$. As $\Omega_{\infty} \subset H_{i+1}$ for all $i$, the sets $\Omega_{\infty} \cap \Omega_{i}, \Omega_{\infty} \cap \tilde{\Omega}_{i}$ and $\Omega_{\infty} \cap \tilde{\Omega}_{\infty}$ are at most finite. If there are only finitely many $M_{i}$, we set $\Omega_{\infty}=\varnothing$.

We need one more definition. Let $Z_{i}$ be the set of all $\mathrm{x} \in \mathrm{bd} F_{i} \mid \mathrm{bd} G_{i}$ (bd means boundary) such that there is a $k$ with $\sigma^{m} \mathrm{x} \in \mathrm{bd} G_{i}$ for all $m \geqslant k$ and of all $\mathrm{x} \in$ bd $G_{i} \backslash$ bd $F_{i+1}$ such that there is a $k$ with $\sigma^{m} \mathrm{x} \in$ bd $F_{i+1}$ for all $m \geq k$. If $G_{\infty} \neq \varnothing$, let $Z_{\infty}$ be the set of all $\mathrm{x} \in \bigcap_{j \geq i} \mathrm{bd} F_{j} \mid \mathrm{bd} G_{\infty}$ for some $i$ such that there is a $k$ with $\sigma^{m} \mathrm{x} \in$ bd $G_{\infty}$ for all $m \geq k$.

Now we have the following result. 
LEMMA 2. The set $\Omega$ of non-wandering points of $\Sigma_{f}^{+}$is contained in

$$
\bigcup_{1 \leq i \leq \infty} \Omega_{i} \cup \bigcup_{0 \leq i \leq \infty} \tilde{\Omega}_{i} \cup \bigcup_{0 \leq i \leq \infty} Z_{i}
$$

Proof. If $\mathrm{x} \in \bar{F}_{i}$ for all $i$ and there are infinitely many $M_{i} \mathrm{~s}$, then $\mathrm{x} \in H \cup G_{\infty}$. We consider this case below. As $G_{0}=\Sigma_{f}^{+}$, we find otherwise an $i<\infty$ with $\mathrm{x} \in \bar{F}_{i} \backslash \bar{G}_{i}$ or $\mathbf{x} \in \bar{G}_{i} \backslash \bar{F}_{i+1}$. We consider first the case $\mathbf{x} \in \bar{F}_{i} \backslash \bar{G}_{i}$. The following three possibilities can occur.

(i) $\mathrm{x} \in \Omega_{i}$.

(ii) $\mathbf{x} \notin \Omega_{\mathrm{i}}$ and $\mathrm{x} \in \operatorname{int}\left(F_{i} \backslash G_{i}\right)$ (int means interior). As $\mathrm{x} \notin \Omega_{i}$, there is a $k$ with $\mathbf{x} \notin \overline{\sigma^{-k}\left(F_{i} \backslash G_{i}\right)}$. Since this set is closed, there is a neighbourhood $U$ of $\mathbf{x}$ contained in int $\left(F_{i} \mid G_{i}\right) \cap{ }_{0}\left[x_{0} \cdots x_{k}\right]$ such that $U \cap \sigma^{-k}\left(F_{i} \mid G_{i}\right)=\varnothing$. But then $\sigma^{k}(U) \cap$ $F_{i} \backslash G_{i}=\varnothing$ and $\sigma^{k}(U) \subset G_{i}$, because $\sigma\left(F_{i}\right) \subset F_{i}$. Because $\sigma\left(G_{i}\right) \subset G_{i}$, we have $\sigma^{m}(U) \subset G_{i}$ for all $m \geq k$. This means that $\mathbf{x}$ is wandering, i.e. $\mathbf{x} \notin \Omega$.

(iii) $\mathbf{x} \notin \Omega_{i}$ and $\mathbf{x} \in$ bd $F_{i} \backslash$ bd $G_{i}$ As $\mathbf{x} \notin \Omega_{i}$, there is a $k$ with $\mathbf{x} \notin \sigma^{-k}\left(\overline{F_{i} \backslash G_{i}}\right)$, i.e. $\sigma^{k} \mathbf{x} \notin \bar{F}_{i} \backslash G_{i}$, hence $\sigma^{k} \mathbf{x} \in G_{i}$. If $\sigma^{m} \mathbf{x} \in$ int $G_{i}$ for some $m$, then there is a neighbourhood $U$ of $\mathbf{x}$ with $U \cap G_{i}=\varnothing$ and $\sigma^{m}(U) \subset G_{i}$, because $\sigma$ is continuous. Because $\sigma\left(G_{i}\right) \subset$ $G_{i}$, we then have $\mathbf{x} \notin \Omega$. If $\sigma^{m} \mathbf{x} \in$ bd $G_{i}$ for all $m \geq k$, then $\mathbf{x} \in Z_{i}$.

Now we consider the case $\mathbf{x} \in \bar{G}_{i} \backslash \bar{F}_{i+1}$. We have the same three possibilities.

(i) $\mathbf{x} \in \tilde{\Omega}_{i}$.

(ii) $\mathbf{x} \notin \tilde{\Omega}_{i}$ and $\mathbf{x} \in$ int $\left(G_{i} \backslash F_{i+1}\right)$. As above, it follows that $\mathbf{x} \notin \Omega$.

(iii) $\mathbf{x} \notin \tilde{\Omega}_{i}$ and $\mathbf{x} \in$ bd $G_{i} \backslash$ bd $F_{i+1}$. As above, we have either $\mathbf{x} \notin \Omega$ or $\mathbf{x} \in Z_{i}$.

If now $\mathrm{x} \in \bar{F}_{i}$ for all $i$ and there are infinitely many $M_{i}$, then $\mathrm{x} \in H \cup G_{\infty}$. We consider again the three possibilities as above.

(i) $x \in \Omega_{\infty} \cup \tilde{\Omega}_{\infty}$.

(ii) $\mathbf{x} \notin \Omega_{\infty} \cup \tilde{\Omega}_{\infty}$ and $\mathbf{x} \in$ int $\left(F_{i} \backslash G_{\infty}\right)$ for all $i$. As

$$
\Omega_{\infty}=\bigcap_{k=0}^{\infty} \sigma^{-k}\left(\bigcap_{i=0}^{\infty} H_{i}\right)=\bigcap_{i=0}^{\infty} \bigcap_{k=0}^{\infty} \sigma^{-k}\left(H_{i}\right),
$$

there is an $i$ with $\mathbf{x} \notin \bigcap_{k=0}^{\infty} \sigma^{-k}\left(H_{i}\right)$. As $\mathbf{x} \in$ int $H_{i}$, it follows as above that $\mathbf{x} \notin \Omega$.

(iii) $\mathbf{x} \notin \Omega_{\infty} \cup \tilde{\Omega}_{\infty}$ and $\mathrm{x} \in \mathrm{bd} F_{i}$ for some $i$. Because $F_{j+1} \subset F_{j}$ and $\mathbf{x} \in \bar{F}_{j}$ for all $j$, one has from $\mathrm{x} \in \mathrm{bd} F_{i}$ that $\mathrm{x} \in \mathrm{bd} F_{j}$ for all $j \geq i$. Then it follows again as above that either $\sigma^{m} \mathbf{x} \in$ int $G_{\infty}$ for some $m$, and hence $\mathrm{x} \in \Omega$ (note that $\mathbf{x} \notin \bar{G}_{\infty}=\tilde{\Omega}_{\infty}$ ), or $\mathbf{x} \in Z_{\infty}$.

In any case, we have shown that either $\mathrm{x} \notin \Omega$ or $\mathrm{x} \in \bigcup \Omega_{i} \cup \cup \tilde{\Omega}_{i} \cup \cup Z_{i}$. This proves the lemma.

Examples which show how the $\Omega_{i}$ look like can be found in [3]. We give here an example where a $Z_{i}$ occurs. The transformation $f$ on $[0,1]$ shown in figure 1 is a modification of an example given by L. Block and L. S. Young (cf. [7]). We have $0<x<d<c<p<e<1, f$ is increasing on the intervals $[0, d),[d, c),[c, e)$ and $[e, 1]$, and it satisfies

$$
f([0, d))=[c, 1), \quad f([d, c))=[x, 1), \quad f([c, e))=[x, 1) \text { and } f([e, 1])=[p, 1] .
$$

Furthermore, $f(x)=f(p)=p$.

The diagram $M$ is as follows (we take the elements of $D$ here as sub-intervals of $[0,1])$. 


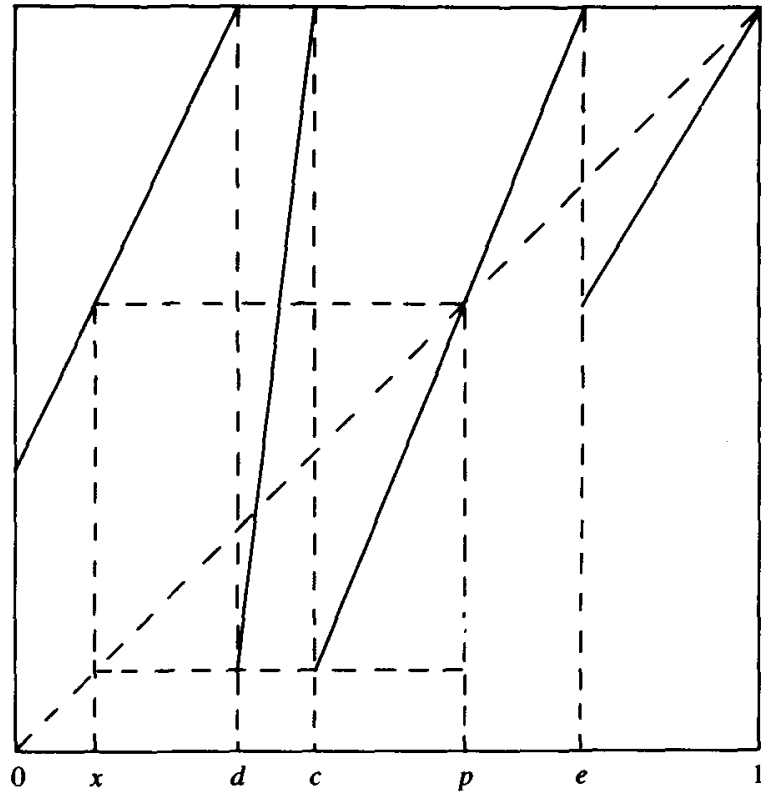

FIGURE 1

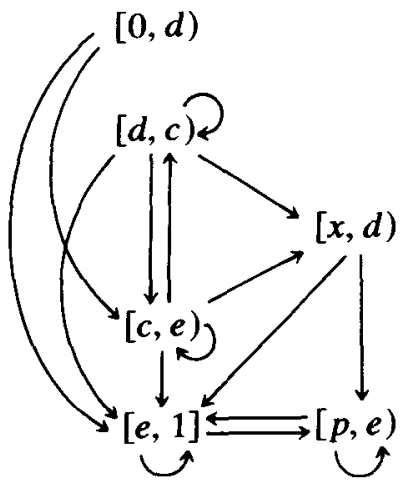

$$
\begin{aligned}
& D_{1}=\{[d, c),[c, e)\} \\
& D_{2}=\{[e, 1],[p, e)\} \\
& \bar{D}_{1}=D_{1} \cup D_{2} \cup\{[x, d)\} \\
& D_{1}^{\prime}=D_{2} \cup\{[x, d)\} \\
& \bar{D}_{2}=D_{2} \\
& D_{2}^{\prime}=\varnothing
\end{aligned}
$$

$G_{0}=[0,1], \quad F_{1}=[x, 1], \quad G_{1}=[x, d) \cup[p, 1], \quad F_{2}=[p, 1], \quad G_{2}=\varnothing . \quad \check{\Omega}_{0}=\varnothing$, $\Omega_{1}=\bigcap_{k=0}^{\infty} f^{-k}([d, p]), \quad$ a Cantor set, $\tilde{\Omega}_{1}=\varnothing, \quad \Omega_{2}=[p, 1] . \quad Z_{1}=\{x\}$ and $\Omega=$ $\Omega_{1} \cup \Omega_{2} \cup\{x\}$.

One sees that $x \notin \Omega_{1}$ and $x \notin \Omega_{2}$. Hence the $Z_{i}$ in lemma 2 are necessary.

Before we are able to investigate $\Omega_{i}$ and $\tilde{\Omega}_{i}$ further, we need two technical lemmas.

LeMMA 3. Let $d \in D$. Then $\mathrm{x} \in d$, if and only if $\mathbf{x}$ can be represented as a path in $M$, which begins with an arrow ending at $d$. 
Proof. Suppose $d=\sigma^{k}\left({ }_{0}\left[a_{0}^{i} \cdots a_{k}^{i}\right]\right)$. Let $\mathbf{x} \in d$. This means $\mathbf{y}=a_{0}^{i} \cdots a_{k-1}^{i} x_{0} x_{1} \cdots \epsilon$ $\Sigma_{f}^{+}$. By the 1-1 correspondence of points in $\Sigma_{f}^{+}$and special paths in $M$ (cf. $\S 1$ ), we have that $\mathbf{y}$ corresponds to the special path

$$
\stackrel{a_{0}^{i}}{\longrightarrow}\left[a_{0}^{i}\right] \stackrel{a_{1}^{i}}{\longrightarrow} \sigma\left({ }_{0}\left[a_{0}^{i} a_{1}^{i}\right]\right) \stackrel{a_{2}^{i}}{\longrightarrow} \cdots \stackrel{x_{0}}{\longrightarrow} \sigma^{k}\left({ }_{0}\left[a_{0}^{i} \cdots a_{k}^{i}\right]\right) \stackrel{x_{1}}{\longrightarrow} \cdots
$$

in $M$. This means $\mathrm{x}$ can be represented as a path in $M$ beginning with an arrow which ends at $d$. Now let $\mathbf{x}$ correspond to a path which begins with an arrow ending at $d$. Then $a_{0}^{i} \cdots a_{k-1}^{i} x_{0} x_{1} \cdots \in \Sigma_{f}^{+}$because it corresponds to a special path as above. Hence $\mathbf{x} \in \sigma^{k}\left({ }_{0}\left[a_{0}^{i} \cdots a_{k}^{i}\right]\right)=d$.

LEMMA 4. Let $\mathbf{y}^{1}, \mathbf{y}^{2} \in \Sigma_{M}$. If $\chi\left(\mathbf{y}^{1}\right)=\chi\left(\mathbf{y}^{2}\right)=\mathbf{x} \notin E$, where $E=\left\{\sigma^{m} \mathbf{a}^{i}, \sigma^{m} \mathbf{b}^{i}: 1 \leq i \leq n\right.$, $m \geq 0\}$, then there is a $K$ with $y_{t}^{1}=y_{t}^{2}$ for all $t \geq K$.

Proof. Suppose $y_{0}^{1}$ is the interval $\left[\sigma^{k} \mathbf{a}^{i}, \sigma^{k^{\prime}} \mathbf{b}^{i^{\prime}}\right]$. Using (1.3) one can determine what $y_{1}^{1}$ is. If $x_{1}=a_{k+1}^{i}$, then $y_{1}^{1}$ has initial point $\sigma^{k+1} a^{i}$. If $x_{1} \neq a_{k+1}^{i}$, then $y_{1}^{1}$ has initial point $\mathbf{a}^{i_{2}}$, where $i_{2}=x_{1}$. Proceeding in this way, we can determine the initial points of $y_{t}^{1}$ for $t \geq 0$. We obtain the following result. Determine $r_{1}, r_{2}, \ldots\left(r_{l} \geq 1\right)$ and $i_{1}=i, i_{2}, \ldots\left(1 \leq i_{l} \leq n\right)$ inductively according to

$$
\begin{array}{ll}
a_{k+t}^{i_{1}}=x_{t} & \text { for } 0 \leq t<r_{1}, \quad a_{k+r_{1}}^{i_{1}} \neq x_{r_{1}}, \\
a_{t-R_{l}}^{i_{l+1}}=x_{t} & \text { for } R_{l} \leq t<R_{l+1}, \quad a_{r_{l+1}}^{i_{l+1}} \neq x_{R_{l+1}} \quad(l \geq 1),
\end{array}
$$

where we have written $R_{t}$ for $r_{1}+\cdots+r_{t}$. Then it follows from (1.3) that the initial point of $y_{t}^{1}$ is $\sigma^{k+t} \mathbf{a}^{i_{1}}$ for $0 \leq t<r_{1}$ and $\sigma^{t-R_{l}} \mathbf{a}^{i_{l+1}}$ for $R_{l} \leq t<R_{l+1}(l \geq 1)$.

We do the same for $\mathbf{y}^{2}$. Suppose $y_{0}^{2}=\left[\sigma^{m} \mathbf{a}^{j}, \sigma^{m^{\prime}} \mathbf{b}^{j^{\prime}}\right]$. We determine $s_{1}, s_{2}, \ldots\left(s_{l} \geq\right.$ 1) and $j_{1}=j, j_{2}, \ldots\left(1 \leq j_{l} \leq n\right)$ inductively according to

$$
\begin{array}{lll}
a_{m+t}^{j_{1}}=x_{t} & \text { for } 0 \leq t<s_{1}, & a_{m+s_{1}}^{j_{1}} \neq x_{s_{1}}, \\
a_{t-S_{l}}^{j_{l+1}}=x_{t} & \text { for } S_{l} \leq t<S_{l+1}, & a_{s_{l+1}}^{i_{l+1}} \neq x_{S_{l+1}}
\end{array} \quad(l \geq 1),
$$

where we have written $S_{t}$ for $s_{1}+\cdots+s_{t}$. By (1.3) we have that the initial point of $y_{t}^{2}$ is $\sigma^{m+t} \mathbf{a}^{j_{1}}$ for $0 \leq t<s_{1}$ and $\sigma^{t-S_{l}} \mathbf{a}^{j_{l+1}}$ for $S_{l} \leq t<S_{l+1}(l \geq 1)$.

Without loss of generality, we can assume that $r_{1} \leq s_{1}$. Because $\mathbf{x} \notin E$, we have $s_{1}<\infty$. If $r_{1}<s_{1}$, it follows then from lemma 4 of [3] that there is a $u$ with $r_{1}+\cdots+r_{u}=s_{1}$. If $r_{1}=s_{1}$, we set $u=1$. Now it follows from (2.4) and (2.5) that $r_{u+l}=s_{1+l}$ and $i_{u+l}=j_{1+l}$ for $l \geq 1$. Hence $y_{t}^{1}$ and $y_{t}^{2}$ have the same initial points for all $t \geq s_{1}:=K^{\prime}$.

We can perform the same also for endpoints of $y_{t}^{1}$ and $y_{t}^{2}$ and find a $K^{\prime \prime}$ such that $y_{t}^{1}$ and $y_{t}^{2}$ have the same endpoints for all $t \geq K^{\prime \prime}$. If one now sets $K=$ $\max \left(K^{\prime}, K^{\prime \prime}\right)$, one has $y_{t}^{1}=y_{t}^{2}$ for all $t \geq K$ and the lemma is proved.

Remarks. (i) Lemma 4 asserts a kind of injectivity of $\chi$. In particular, if $y^{1}$ and $y^{2}$ are distinct periodic points in $\Sigma_{M} \backslash \chi^{-1}(E)$, then $\chi\left(\mathbf{y}^{1}\right) \neq \chi\left(\mathbf{y}^{2}\right)$.

(ii) The proof of lemma 4 works also if one has instead of $y^{1}$ and $y^{2}$ only one-sided paths $y_{0}^{1} y_{1}^{1} y_{2}^{1} \cdots$ and $y_{0}^{2} y_{1}^{2} y_{2}^{2}$ in $M$, which correspond to the same $\mathbf{x} \in \Sigma_{f}^{+} \backslash E$. We shall sometimes apply lemma 4 in this form.

We return to the investigation of $\Omega_{i}$ and $\tilde{\Omega}_{i}$. 
LEMMA 5. If $\Sigma_{M_{i}}$ does not consist of only a periodic orbit, then $\chi\left(\Sigma_{M_{i}}\right) \subset \Omega_{i}$.

Proof. Let $\mathbf{y}=\cdots d_{0} d_{1} d_{2} \cdots \in \Sigma_{M_{i}}$ and suppose $\sigma^{k} \mathbf{x} \notin E$ for $k \geq 0$, where $\mathbf{x}=\chi(\mathbf{y})$. By definition of $\chi$ and lemma 3 , we have $\mathrm{x} \in d_{0} \in D_{i}$, hence $\mathrm{x} \in F_{i}$. We show $\mathrm{x} \notin G_{i}$. If $\mathbf{x} \in G_{i}$, then $\mathbf{x} \in d_{0}^{\prime}$ for some $d_{0}^{\prime} \in D_{i}^{\prime}$. By lemma 3 , there is a path in $M$, beginning with an arrow ending at $d_{0}^{\prime}$, which corresponds to $\mathbf{x}$. Let $d_{0}^{\prime} d_{1}^{\prime} d_{2}^{\prime} \cdots$ be the vertices on this path. By lemma 4 there is a $K>0$ such that $d_{k}=d_{k}^{\prime}$ for $k \geq K$, since $\mathrm{x} \notin E$. As $d_{k} \in D_{i}$, we have found a path from $D_{i}^{\prime}$ to $D_{i}$, a contradiction to the definition of $D_{i}^{\prime}$. Hence $\mathbf{x} \in F_{i} \backslash G_{i}$. But also $\sigma^{k}(\mathbf{x})=\chi\left(\sigma^{k} \mathbf{y}\right) \notin E$ and therefore, as for $\mathbf{x}$ above, it follows that $\sigma^{k} \mathbf{x} \in F_{i} \backslash G_{i}$ for all $k \geq 0$. This implies $\mathbf{x} \in \Omega_{i}$.

Now suppose that $\sigma^{k} \mathbf{x} \in E$ for some $k$. As $\Sigma_{M_{i}}$ does not consist of only a periodic orbit, we can find $\mathbf{y}^{n} \in \Sigma_{M_{i}}$ with $\sigma^{k}\left(\chi\left(\mathbf{y}^{n}\right)\right) \notin E$, for all $k$, converging to $\mathbf{y}$ in $\Sigma_{M_{i}}\left(M_{i}\right.$ is irreducible). As $\chi$ is continuous and $\Omega_{i}$ is closed, it follows that $\chi(y)=\lim \chi\left(\mathbf{y}^{n}\right) \epsilon$

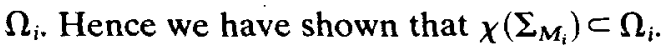

Now set $\tilde{D}_{i}=D_{i}^{\prime} \backslash \bar{D}_{i+1}, \tilde{D}_{0}=D \backslash \bar{D}_{1}, \tilde{D}_{\infty}=D_{\infty}^{\prime}$ and $\tilde{M}_{i}=M / \tilde{D}_{i}$ for $0 \leq i \leq \infty$. Let $S_{i} \subset \Sigma_{f}^{+}$be the set of all $\mathrm{x}$ which can be represented as one-sided paths in $\tilde{M}_{i}$. If $\tilde{D}_{i}$ is finite, then $S_{i}=\varnothing$, because $\tilde{M}_{i}$ contains no closed paths. All closed paths must be contained in the $M_{i}$ s by definition.

We have now:

LEMMA 6. $\Omega_{i} \subset \overline{\chi\left(\bar{\Sigma}_{\left.M_{i}\right)}\right.}$ for $i<\infty, \Omega_{i} \subset \bar{S}_{i}$ for $i \leq \infty$ and the set $\Omega_{i} \backslash \chi\left(\Sigma_{M_{i}}\right)$ is at most countable and contains only finitely many periodic points.

Proof. We give the proof only for $\Omega_{i}$. It is the same for $\tilde{\Omega}_{i}$. First we show that $\chi\left(\Sigma_{M_{i}}\right)$ is dense in $\Omega_{i}$. Suppose ${ }_{0}\left[x_{0} \cdots x_{k-1}\right] \cap \Omega_{i} \neq \varnothing$. Because ${ }_{0}\left[x_{0} \cdots x_{k-1}\right]$ is open, there is at least one $d \in D_{i}$ with $0\left[x_{0} \cdots x_{k-1}\right] \cap d \neq \varnothing$. For every such $d, x_{0} \cdots x_{k-1}$ corresponds to a path of length $k$ beginning with an arrow ending at $d$ (lemma 3 ). If all these paths leave $M_{i}$, we have

$$
{ }_{0}\left[x_{0} \cdots x_{k-1}\right] \cap\left(F_{i} \backslash \bigcup_{m=0}^{k-1} \sigma^{-m}\left(G_{i}\right)\right)=\varnothing .
$$

As ${ }_{0}\left[x_{0} \cdots x_{k-1}\right]$ is open, this implies $0\left[x_{0} \cdots x_{k-1}\right] \cap \Omega_{i}=\varnothing$, a contradiction. Hence we have a path of length $k$ in $M_{i}$ which corresponds to $x_{0} \cdots x_{k-1}$. Let $d_{0} \cdots d_{k-1}$ be the vertices on this path. As $M_{i}$ is irreducible, we can extend $d_{0} \cdots d_{k-1}$ to a two-sided path in $M_{i}$ giving rise to a $\mathbf{y} \in \Sigma_{M_{i}}$. By definition of $\chi, \chi(\mathbf{y}) \in_{0}\left[x_{0} \cdots x_{k-1}\right]$. (In the case of $\tilde{\Omega}_{i}$ we extend $d_{0} \cdots d_{k-1}$ to a one-sided path in $\tilde{D}_{i}$ giving rise to an element of $S_{i}$.) This proves that $\Omega_{i} \subset \overline{\chi\left(\Sigma_{M_{i}}\right)}$.

Now let $V$ be the set of all $\mathrm{x} \in \Omega_{i}$ with $\sigma^{k} \mathbf{x} \in E$ for some $k$ or with $\mathbf{x} \in$ bd $F_{i}$. Then $V$ is countable and contains at most finitely many periodic points. In order to show the second assertion it suffices to prove that $\Omega_{i} \backslash V \subset \chi\left(\Sigma_{M_{i}}\right)$.

Let $\mathbf{x} \in \Omega_{i} \backslash V$. As $\mathbf{x} \notin$ bd $F_{i}$ there is a $d_{0} \in D_{i}$ with $\mathbf{x} \in d_{0}$. By lemma 3 there is a path in $M$ which begins with an arrow ending at $d_{0}$ and which corresponds to $\mathbf{x}$. Let $d_{0} d_{1} d_{2} \cdots$ be the vertices on this path. We show that $d_{k} \in D_{i}$. Suppose $d_{k} \notin D_{i}$, then we have $d_{k} \in D_{i}^{\prime}$. By lemma 3 we have $\sigma^{k} \mathbf{x} \in d_{k}$. As the endpoints of the interval $d_{k}$ are in $E$ and $\mathbf{x} \notin V, \sigma^{k} \mathbf{x}$ is in the interior of $d_{k}$. Hence $\sigma^{k} \mathbf{x} \in$ int $G_{i}$, i.e. $\sigma^{k} \mathbf{x} \notin \overline{F_{i} \backslash G_{i}}$, 
a contradiction to $\mathrm{x} \in \Omega_{i}$. Hence $d_{k} \in D_{i}$ for all $k$. Because $M_{i}$ is irreducible, we can extend the path $d_{0} d_{1} d_{2} \cdots$ to a two-sided one in $M_{i}$, which gives a $\mathbf{y} \in \Sigma_{M_{i}}$. By definition of $\chi, \chi(\mathbf{y})=\mathbf{x}$, hence $\mathbf{x} \in \chi\left(\Sigma_{M_{i}}\right)$. This proves that $\Omega_{i} \backslash V \subset \chi\left(\Sigma_{M_{i}}\right)$.

Remarks. (i) If $\Sigma_{M_{i}}$ is only a periodic orbit, then $\Omega_{i}=\chi\left(\Sigma_{M_{i}}\right)$ or is empty. In this case we redefine $\Omega_{i}$ as $\chi\left(\Sigma_{M_{i}}\right)$. Then we have $\Omega_{i}=\overline{\chi\left(\Sigma_{\left.M_{i}\right)}\right.}$ for all $i$ by lemmas 5 and 6 .

(ii) If $D_{i}$ is finite, then $\Sigma_{M_{i}}$ is compact. As $\chi$ is continuous, this implies that $\chi\left(\Sigma_{M_{i}}\right)$ is closed. Hence $\chi\left(\Sigma_{M_{i}}\right)=\Omega_{i}$. An open question is whether this also happens if $D_{i}$ is infinite.

(iii) If $\tilde{D}_{i}$ is finite, then $S_{i}=\varnothing$. By lemma 6 we then have $\tilde{\Omega}_{i}=\varnothing$.

The next two lemmas give properties of $\Omega_{i}$ for $i \neq \infty$.

LEMMA 7. (i) $\sigma \mid \Omega_{i}$ is topologically transitive.

(ii) If $\mathbf{x} \in \Sigma_{f}^{+}$is periodic, then $\mathbf{x} \in \Omega_{i}$ for some $i$.

Proof. (i) Because $M_{i}$ is irreducible, we can find a $\mathbf{y} \in \Sigma_{M_{i}}$ such that $\left\{\sigma^{k} \mathbf{y}: k \geq 0\right\}$ is dense in $\Sigma_{M_{i}}$. As $\chi$ is continuous and commutes with $\sigma$, the set $\left\{\sigma^{k} \mathbf{x}: k \geq 0\right\}$, where $\mathbf{x}=\chi(\mathbf{y})$, is dense in $\Omega_{i}$ by lemma 6 .

(ii) Let $\mathbf{x} \in \Sigma_{f}^{+}$satisfy $\sigma^{p} \mathbf{x}=\mathbf{x}$ for some $p$. Choose a path $y_{0} y_{1} \cdots$ in $M\left(y_{i} \in D\right)$ which corresponds to $\mathbf{x}$. Suppose first that $\mathbf{x} \notin E$. Because $\sigma^{p} \mathbf{x}=\mathbf{x}$, the path $y_{p} y_{p+1} \cdots$ also corresponds to $\mathbf{x}$. Hence it follows from lemma 4 that there is a $K$ with $y_{m+p}=y_{m}$ for all $m \geq K$. Now suppose that $\mathbf{x} \in E$, say $\mathbf{x}=\sigma^{k} \mathbf{b}^{j}$. Set $y_{m}=$ $\sigma^{k+m}\left({ }_{0}\left[b_{0}^{i} \cdots b_{k+m}^{i}\right]\right)$. Then $y_{0} y_{1} \cdots$ is a path in $M$ which corresponds to $\mathbf{x}$. As in the proof of lemma 4 define $r_{1}, r_{2}, \ldots\left(r_{l} \geq 1\right)$ and $i_{1}, i_{2}, \ldots$ inductively such that, for $l \geq 0$,

$$
a_{t-R_{l}}^{i_{l+1}}=b_{t}^{j} \quad \text { for } R_{l} \leq t<R_{l+1}, \quad a_{r_{l+1}}^{i_{l+1}} \neq b_{R_{l+1}}^{j},
$$

where we have written $R_{m}$ for $r_{1}+\cdots+r_{m}\left(R_{0}=0\right)$. It follows again from (1.3) that $y_{m}=\left[\sigma^{k+m-R_{l}} \mathbf{a}^{i_{i+1}}, \sigma^{k+m} \mathbf{b}^{j}\right]$, where $l$ is such that $R_{l} \leq k+m<R_{l+1}$.

If $r_{t}=\infty$ for some $t$, then $y_{m}=\left\{\sigma^{k+m} \mathbf{b}^{j}\right\}$ for $m \geq R_{t-1}-k=: K$ and hence $y_{m+p}=y_{m}$ for all $m \geq K$. If $r_{l}<\infty$ for all $l$, let $u$ and $v$ be such that $R_{u-1}<k \leq R_{u}$ and $R_{v-1}<k+p \leq R_{v}$. If $R_{u}+p<R_{v}$ it follows from $\sigma^{k+p} \mathbf{b}^{j}=\sigma^{k} b^{j}$ and lemma 4 of [3] that there is a $w>u$ with $\boldsymbol{R}_{w}+p=\boldsymbol{R}_{v}$. If $\boldsymbol{R}_{u}+p>\boldsymbol{R}_{v}$, we can increase $v$ to obtair equality. Hence it follows from (2.6) that $r_{w+l}=r_{v+l}$ and $i_{w+l}=i_{v+l}$ for all $l \geq 1$. This implies $y_{m+p}=y_{m}$ for all $m \geq R_{w}-k=: K$.

Hence in any case we have found a closed path $y_{K} \rightarrow y_{K+1} \rightarrow \cdots \rightarrow y_{K+p}=y_{K}$ in $M$, which must then belong to some $M_{i}$ by definition of the $M_{j} \mathrm{~s}$. As $\mathrm{x}$ is the point which corresponds to this closed path, we have by lemma 5 that $\mathbf{x} \in \Omega_{i}$ (cf. also the remark after lemma 6).

LEMMA 8. (i) If $D_{i}$ is maximal with respect to (2.2) and $\tilde{D}_{i}=\varnothing$, then $\Omega_{i}$ is a finite union of intervals.

(ii) If $\Sigma_{M_{i}}$ is a periodic orbit, $\Omega_{i}$ is also a periodic orbit.

(iii) Otherwise, $\Omega_{i}$ is a Cantor set. 
Proof. (i) Let $\mathbf{x} \in F_{i} \backslash G_{i}$. Then $\mathbf{x} \in d$ for some $d \in D_{i}$. By lemma 3, $\mathbf{x}$ corresponds to a path which begins with an arrow ending at $d$. Because $D_{i}$ is maximal and $\tilde{D}_{i}=\varnothing$, this path cannot leave $\boldsymbol{M}_{i}$. Extending it to a two-sided path gives a $\mathbf{y} \in \Sigma_{M_{i}}$ with $\chi(\mathbf{y})=\mathbf{x}$. Hence $\Omega_{i}=\overline{\chi\left(\Sigma_{M_{i}}\right)}=\overline{F_{i} \backslash G_{i}}$, a finite union of intervals.

(ii) We have $\Omega_{i}=\chi\left(\Sigma_{M_{i}}\right)$ by definition (cf. the remark after lemma 6).

(iii) The set $\bigcap_{k=0}^{m} \overline{\sigma^{-k}\left(F_{i} \backslash G_{i}\right)}$ is a finite union of closed intervals. The intersection of these sets for $m \geq 1$ gives $\Omega_{i}$. Furthermore, $\Omega_{i}$ cannot contain an interval. Suppose ${ }_{0}\left[x_{0} \cdots x_{k-1}\right] \subset \Omega_{i}$. Then one finds a path of length $k$ in $M_{i}$ which corresponds to $x_{0} \cdots x_{k-1}$. We can extend this path to an infinite one which leaves $M_{i}$ and find an $\mathbf{x} \in \in_{0}\left[x_{0} \cdots x_{k-1}\right]$ with $\sigma^{m} \mathbf{x} \in$ int $G_{i}$ for some $m$. Hence $\mathbf{x} \notin \Omega_{i}$, a contradiction to ${ }_{0}\left[x_{0} \cdots x_{k-1}\right] \subset \Omega_{i}$. As $\Sigma_{M_{i}}$ contains uncountably many elements, the same is true for $\Omega_{i}$ by lemmas 5 and 4 . Therefore $\Omega_{i}$ is a Cantor set.

Next we investigate $\tilde{\Omega}_{i}$ for $0 \leq i \leq \infty$.

LEMMA 9. (i) $h_{\text {top }}\left(\tilde{\Omega}_{i}\right)=0$.

(ii) Among the $\tilde{\Omega}_{i}$ for $0 \leq i \leq \infty$ there are only finitely many which are not empty.

Proof. (i) We have shown in lemma 6 that $\tilde{\Omega}_{i} \subset \overline{S_{i}}$. Hence it suffices to show that $\lim _{k \rightarrow \infty}(1 / k) \log N_{k}=0$, where $N_{k}$ is the number of admissible blocks of length $k$ in $\bar{S}_{i}$. But if the block $x_{0} \cdots x_{k-1}$ is admissible in $\bar{S}_{i}$, i.e. $0\left[x_{0} \cdots x_{k-1}\right] \cap \bar{S}_{i} \neq \varnothing$, then we also have ${ }_{0}\left[x_{0} \cdots x_{k-1}\right] \cap S_{i} \neq \varnothing$, because ${ }_{0}\left[x_{0} \cdots x_{k-1}\right]$ is open. Hence $N_{k}$ is also the number of admissible blocks of length $k$ in $S_{i}$. Furthermore, we can suppose that $G_{i}$ is all of $\Sigma_{f}^{+}$. Otherwise we restrict $f$ to $\phi^{-1}\left(G_{i}\right)$, which again gives a piecewise monotonic transformation. Then $N_{k}$ is the number of special paths of length $\boldsymbol{k}$ in $\tilde{\boldsymbol{M}}_{i}$.

Fix some $\varepsilon>0$. By lemma 13 of [3] there is a finite subset $A$ of $\tilde{D}_{i}$ such that the spectral radius $r\left(\tilde{M}_{i} / B\right)$ of $\tilde{M}_{i}$ restricted to $B=\tilde{D}_{i} \backslash A$ is less than $\varepsilon$. This implies that the number of paths of length $l$ in $\tilde{M}_{i} / B$ which begin with one of the finitely many arrows leading from $A$ to $B$, is less than $C \exp (2 \varepsilon l)$ for some constant $C$ (cf. $\S 3$ of [3]). Making $A$ larger if necessary, we can also suppose that the finitely many elements of $\tilde{D}_{i}$ at which special paths begin are contained in $A$.

Now let $K$ be the cardinality of $A$. In a special path of length $k$ in $\tilde{M}_{i}$ every element of $\tilde{D}_{i}$ can occur at most once, because $\tilde{M}_{i}$ contains no closed paths. Hence this special path contains at most $K$ blocks consisting of elements of $A$, each of which has length at most $K$. One of these blocks is at the beginning of the special path. Hence there are not more than $\left(\begin{array}{l}k \\ K\end{array}\right)$ possibilities to choose the places of these blocks in the special path of length $k$. In between there are blocks consisting of elements of $B$ which begin with an arrow leading from $A$ to $B$. They have lengths $l_{1}, l_{2}, \ldots, l_{j}(j \leq K)$ with $\sum_{i=1}^{j} l_{i} \leq k$. Hence we have

$$
N_{k} \leq\left(\begin{array}{c}
k \\
K
\end{array}\right) n^{K^{2}} \cdot C \exp \left(2 \varepsilon l_{1}\right) \cdots C \exp \left(2 \varepsilon l_{j}\right) \leq\left(\begin{array}{c}
k \\
K
\end{array}\right) n^{K^{2}} \cdot C^{j} \exp (2 \varepsilon k) .
$$


This implies that $\lim (1 / k) \log N_{k} \leq 2 \varepsilon$. As $\varepsilon$ was arbitrary, we obtain $\lim (1 / k) \log N_{k}=0$.

(ii) If $\tilde{D}_{i}$ is finite, then $S_{i}=\varnothing$, and hence $\tilde{\Omega}_{i}=\varnothing$ by lemma 6 . If $\tilde{D}_{i}$ is infinite, then it contains a set of the form

$$
\left\{\sigma^{k}\left({ }_{0}\left[a_{0}^{i} \cdots a_{k}^{i}\right]\right): k \geq m\right\} \quad \text { or } \quad\left\{\sigma^{k}\left({ }_{0}\left[b_{0}^{i} \cdots b_{k}^{i}\right]\right): k \geq m\right\} \text { for some } i \text { and } m,
$$

because $D_{i}^{\prime}$ and $\bar{D}_{i+1}$ have the property (2.3) which implies that $\tilde{D}_{i}$ satisfies (2.1). Hence there can be at most $2 n$ different $\tilde{D}_{i}$, which contain infinitely many elements, and hence at most $2 n$ different non-empty $\tilde{\Omega}_{i}$.

Remark. The transformation $x \rightarrow x+\alpha(\bmod 1)$ on $[0,1], \alpha \notin \mathbb{Q}$, is an example where $\tilde{D}_{0}=D$ and $\tilde{\Omega}_{0}=\Sigma_{f}^{+}$. It would be interesting either to find an example where $\tilde{\Omega}_{i} \neq \varnothing$ for some $i$ such that there is a $j>i$ with $D_{j} \geq D_{i}$, or to show that this cannot happen. LEMMA 10. If there are infinitely many $M_{i}, s_{\text {, }}$ then $h_{\text {top }}\left(\bigcap_{i=1}^{\infty} \bar{F}_{i}\right)=0$. In particular, $h_{\text {top }}\left(\Omega_{\infty}\right)=0$.

Proof. Fix some $\varepsilon>0$. We have $\bar{D}_{i}=\left(\bar{D}_{i} \mid D_{\infty}^{\prime}\right) \cup D_{\infty}^{\prime}$. As $\bigcap_{i=1}^{\infty}\left(\bar{D}_{i} \mid D_{\infty}^{\prime}\right)=\varnothing$, there is an $i$ with $r\left(L_{i}\right)<\exp \varepsilon$, where $L_{i}=M /\left(\bar{D}_{i} \mid D_{\infty}^{\prime}\right)$, by lemma 13 of [3].

Let $x_{0} x_{1} \cdots x_{k-1}$ be an admissible block of length $k$ in $\bar{F}_{i}$. It follows from the proof of lemma 1 that $F_{i}$ is a finite union of intervals

$$
\sigma^{m} A_{i}=\bigcup_{r, l}\left(\left[\sigma^{m} \mathbf{a}^{j}, \sigma^{m+l} \mathbf{b}^{r}\right]\right) \text { and } \sigma^{m} B_{j}=\bigcup_{r, l}\left(\left[\sigma^{m+l} \mathbf{a}^{r}, \sigma^{m} \mathbf{b}^{j}\right]\right)
$$

If ${ }_{0}\left[x_{0} \cdots x_{k-1}\right] \cap \overline{\sigma^{m} A_{j}} \neq \varnothing$, then there are $r$ and $l$ such that

$$
\left[\boldsymbol{\sigma}^{m} \mathbf{a}^{i}, \sigma^{m+l} \mathbf{b}^{r}\right] \cap_{0}\left[x_{0} \cdots x_{k-1}\right] \neq \varnothing .
$$

By lemma 3, $x_{0} \cdots x_{k-1}$ can then be represented as a path of length $k$ in $M / \bar{D}_{i}$ which begins at $\left[\sigma^{m} \mathbf{a}^{j}, \sigma^{m+l} \mathbf{b}^{r}\right] \in \bar{D}_{i}$. Let $z$ be the number of intervals of which $F_{i}$ consists. Then for every $k$, we can find $z$ elements $d_{1}, \ldots, d_{z}$ of $\bar{D}_{i}$ such that every admissible block in $\bar{F}_{i}$ of length $k$ can be represented as path of length $k$ in $M / \bar{D}_{i}$ beginning at one of these $z$ elements.

By definition of $D_{\infty}^{\prime}$, no path leads from $D_{\infty}^{\prime}$ to $\bar{D}_{i} \mid D_{\infty}^{\prime}$. Hence for every admissible block $x_{0} \cdots x_{k-1}$ there is an $l(0 \leq l \leq k)$ such that $x_{0} \cdots x_{l-1}$ corresponds to a path in $\bar{D}_{i} \backslash D_{\infty}^{\prime}$ and $x_{l} \cdots x_{k-1}$ to a path in $D_{\infty}^{\prime}$. Hence the number of admissible blocks of length $k$ beginning at some $d_{j}(1 \leq j \leq z)$ is less than $\sum_{l=0}^{k} N_{l}^{\prime} N_{k-l}^{\prime \prime}$, where $N_{l}^{\prime}$ is the number of admissible blocks of length $l$ in $\bar{D}_{i} \mid D_{\infty}^{\prime}$ beginning at $d_{j}$ and $N_{k-l}^{\prime \prime}$ is the maximal number of blocks of length $k-l$ in $D_{\infty}^{\prime}$ beginning at some element of $D_{\infty}^{\prime}$.

Let $u$ be the vector, with index set $\bar{D}_{i} \backslash D_{\infty}^{\prime}$, which has entry 1 at the $d_{i}$ th coordinate and entry 0 otherwise. If $d_{i} \in D_{\infty}^{\prime}$, then $u$ has only zero entries (this corresponds to the case $l=0)$. Then $N_{l}^{\prime}=\left\|L_{i}^{\prime} u\right\|_{1} \leq\left\|L_{i}^{\prime}\right\|_{1} \leq C \exp (2 \varepsilon l)$, for some constant $C$, because $r\left(L_{i}\right)<\varepsilon$. 
In the proof of lemma 9, we have shown that $h_{\text {top }}\left(S_{\infty}\right)=0$. Hence $N_{k-l}^{\prime \prime} \leq$ $C^{\prime} \exp (2 \varepsilon(k-l))$ for some constant $C^{\prime}$. If $N_{k}$ is the number of admissible blocks of length $k$ in $\bar{D}_{i}$, we therefore have $N_{k} \leq z k C C^{\prime} \exp (2 \varepsilon k)$. This implies that $h_{\text {top }}\left(\bar{F}_{i}\right)<$ $2 \varepsilon$ and hence

$$
h_{\mathrm{top}}\left(\bigcap_{i=1}^{\infty} \bar{F}_{i}\right)<2 \varepsilon
$$

As $\varepsilon$ was arbitrary, this gives the desired result.

Remark. An example, where one has infinitely many $M_{i}$ s and $\Omega_{\infty} \neq \varnothing$, is $x \rightarrow$ $a x(1-x)$ on $[0,1]$ for certain values of $a \in[2,4]$.

Let $Z$ be the set of all non-wandering $x \in \bigcup_{0 \leq i<\infty} Z_{i}$ which are not contained in some $\Omega_{i}$ or $\tilde{\Omega}_{i}$.

LEMMA 11. (i) If $\mathbf{x} \in Z$, then $\mathbf{x}$ is an isolated point of $\Omega$ which is not periodic. Hence $\mathbf{x}$ is wandering in $\Omega$.

(ii) $Z$ is finite.

Proof. (i) Let $\mathbf{x} \in Z \cap Z_{i}(i<\infty)$. Then

$$
\mathbf{x} \notin \bar{F}_{i+1} \supset \bigcup_{j>i}\left(\Omega_{j} \cup \tilde{\Omega}_{j} \cup Z_{j}\right)
$$

Also $\mathbf{x} \notin \bigcup_{j \leq i}\left(\Omega_{j} \cup \tilde{\Omega}_{j}\right)$, which is a closed set because $\Omega_{i}$ and $\tilde{\Omega}_{i}$ are closed. By lemma 2 , we have

$$
\Omega \subset \bigcup_{j \leq i}\left(\Omega_{j} \cup \tilde{\Omega}_{j}\right) \cup \bar{F}_{i+1} \cup \bigcup_{j \leq i} Z_{j} .
$$

As $\bigcup_{j \leq i} Z_{i}$ is finite, we find a neighbourhood $U$ of $\mathbf{x}$ such that $U \cap \Omega=\{\mathbf{x}\}$. The non-periodicity of $\mathbf{x}$ follows from the definition of $Z_{i}$.

(ii) It follows from (2.1) that there are only finitely many $D_{i}$, say $D_{i_{1}}, \ldots, D_{i}$, which have infinitely many elements. By the proof of (ii) of lemma 9 , there are only finitely many $\tilde{D}_{i}$, say $\tilde{D}_{j_{1}}, \ldots, \tilde{D}_{j_{s}}$, which have infinitely many elements.

If $D_{i}$ is finite, then $\bigcup\left\{d: d \in D_{i}\right\}$ is already closed, because every $d \in D$ is closed. Hence bd $F_{i} \backslash$ bd $G_{i} \subset \bigcup\left\{d: d \in D_{i}\right\}$ and an $\mathrm{x} \in$ bd $F_{i} \backslash \mathrm{bd} G_{i}$ is at the boundary of some $d \in D_{i}$. But then $\mathbf{x}=\sigma^{k} \mathbf{a}^{m}$ or $\sigma^{k} \mathbf{b}^{m}$ for some $k$ and $m$ (cf. (1.2)). As $\sigma^{l} \mathbf{x}$ is periodic for some $l$, we then have $\sigma^{k+l} \mathbf{a}^{m}$ (or $\sigma^{k+l} \mathbf{b}^{m}$ ) is periodic. Similar arguments apply for a finite $\tilde{D}_{i}$. Hence

$$
\begin{aligned}
& Z \subset\left\{\sigma^{k_{i}} \mathbf{a}^{i}, \sigma^{m_{i}} \mathbf{b}^{j}: 1 \leq i, j \leq n, 0 \leq k_{i} \leq K_{i}-1,0 \leq m_{j} \leq M_{j}-1,\right. \\
&\left.\sigma^{K_{i}} \mathbf{a}^{i} \text { and } \sigma^{M_{i} \mathbf{b}^{j}} \text { are periodic }\right\} \\
& \cup \bigcup_{i=1}^{r} Z_{i_{t}} \cup \bigcup_{t=1}^{s} Z_{j_{t^{*}}}
\end{aligned}
$$

This is a finite set.

We collect the results in the following theorem. 
THEOREM 1. $\Omega=\bigcup_{i \geq 1} \Omega_{i} \cup Y \cup Z$ (finite or countable union) such that:

(i) $\Omega_{i}$ and $Y$ are closed, $\sigma$-invariant sets. $Z$ is finite and wandering in $\Omega$.

(ii) $\Omega_{i} \cap \Omega_{j}$ for $i \neq j$ and $\Omega_{i} \cap Y$ are at most finite. $\Omega_{i} \cap Z=\varnothing, Y \cap Z=\varnothing$.

(iii) All periodic points are contained in $\bigcup_{i \geq 1} \Omega_{i}$.

(iv) $\Omega_{i}$ is topologically transitive. It is either a finite union of intervals, a Cantor set, or a periodic orbit.

(v) $h_{\text {top }}(Y)=0$.

Proof. We set $Y=\left(\bigcup_{0 \leq i \leq \infty} \tilde{\Omega}_{i} \cup \bigcap_{i=1}^{\infty} \bar{F}_{i}\right) \cap \Omega$ if there are infinitely many $M_{i}$ s, and $Y=\bigcup_{0 \leq i \leq \infty} \tilde{\Omega}_{i} \cap \Omega$ if there are finitely many $M_{i}$ s. Because $\Omega_{\infty} \cup Z_{\infty} \subset \bigcap_{i=1}^{\infty} \bar{F}_{i}$, it follows from lemma 2 that $\Omega=\bigcup_{i \geq 1} \Omega_{i} \cup Y \cup Z$. By (ii) of lemma 9, $\bigcup_{0 \leq i \leq \infty} \tilde{\Omega}_{i}$ is a finite union of closed sets, hence $Y$ is closed. $Y$ is $\sigma$-invariant, because $\tilde{\Omega}_{i}, \bar{F}_{i}$ and $\Omega$ are $\sigma$-invariant. Together with lemma 11 , this implies (i). (ii) follows from definitions, because $\bigcup_{0 \leq i \leq \infty} \tilde{\Omega}_{i}$ is a finite union. (iii) and (iv) are lemmas 7 and 8, (v) follows from lemmas 9 and 10 .

Now we investigate $\Omega_{i}$ for some fixed $i \neq \infty$. One says that the $0-1$-matrix $M_{i}$ has period $q$ if $D_{i}=C_{1} \cup C_{2} \cup \cdots \cup C_{q}$ (disjoint) and if $d \in C_{k}$ and $M_{d d^{\prime}}=1$ imply $d^{\prime} \in C_{k+1}$ (we take the indices of the $C_{j}$ s modulo $q$ ). $q$ is taken as large as possible. If $q=1, M_{i}$ is called aperiodic. Set $K_{j}=\left(F_{i} \mid G_{i}\right) \cap \bigcup\left\{d: d \in C_{j}\right\}$.

LEMMA 12. $K_{j}$ is a finite union of intervals. $K_{j} \cap K_{m}(j \neq m)$ is empty or finite.

Proof. One shows that, if $d_{1}, d_{2} \in D_{i}$ and the interval $d_{1} \cap d_{2}$ contains more than one point, then $d_{1}$ and $d_{2}$ are in the same $C_{j}$. This proves both assertions. It proves the first assertion, because it implies that the $A_{i} s$ and $B_{i} s$ in the proof of lemma 1 are subsets of one of the $K_{j}$ s. If $d_{1} \cap d_{2}$ is a non-trivial interval, one can choose $\mathbf{y}^{1}$, $\mathbf{y}^{2} \in \Sigma_{M_{i}}$ with $y_{0}^{1}=d_{1}$ and $y_{0}^{2}=d_{2}$ such that $\chi\left(\mathbf{y}^{1}\right)=\chi\left(\mathbf{y}^{2}\right) \notin E$ (lemma 3 and $E$ is countable). By lemma 4 there is a $t$ with $y_{t}^{1}=y_{t}^{2} \in C_{m}$ for some $m$. Hence $y_{t-i}^{1}$ and $y_{t-i}^{2}$ are in $C_{m-i}$. In particular, $y_{0}^{1}=d_{1}$ and $y_{0}^{2}=d_{2}$ are both in $C_{m-t}$, proving the lemma.

Set $\boldsymbol{X}_{j}=\overline{\boldsymbol{K}_{j} \cap \boldsymbol{\Omega}_{i}}$. We have

THEOREM 2. $\Omega_{i}=\bigcup_{j=1}^{q} X_{j} . X_{j}$ is closed and $X_{j} \cap X_{k}$ is empty or finite $(j \neq k) . \sigma\left(X_{j}\right) \subset$ $X_{j+1} \cdot \sigma^{p}: X_{j} \rightarrow X_{i}$ is topologically mixing.

Proof. It remains to prove the last assertion. $\left(X_{j}, \sigma^{q}\right)$ is an $\Omega_{m}$ for the piecewise monotonic transformation $\left(\phi^{-1}\left(K_{j}\right), f^{q}\right)$. The matrix $\dot{M}$ corresponding to $\left(X_{j}, \sigma^{q}\right)$ can be derived from $M_{i}$ as follows. Set

$$
\tilde{D}=\left\{y_{1} \cdots y_{q}: y_{k} \in C_{j+k-1}, M_{y_{k} y_{k+1}}=1 \text { for } 1 \leq k \leq q-1\right\} .
$$

We have an arrow $y_{1} \cdots y_{q} \rightarrow y_{1}^{\prime} \cdots y_{q}^{\prime}$ in $\tilde{M}$ iff there is an arrow $y_{q} \rightarrow y_{1}^{\prime}$ in $M_{i} . \tilde{M}$ is irreducible because $M_{i}$ is. $\tilde{M}$ is aperiodic. If $\tilde{M}$ has period $\tilde{q}$, then it follows that 
$M_{i}$ has period $q \tilde{q}$. In the next section we shall see that there is a $\sigma$-invariant, mixing measure on $\Sigma_{\tilde{M}}$ which is positive on open sets if $h_{\text {top }}\left(\Sigma_{M_{i}}\right) \neq 0$, i.e. $\Sigma_{M_{i}}$ is not a periodic orbit. It follows from proposition (6.7) of [2] that $\Sigma_{\tilde{M}}$ and hence also $\left(X_{j}, \sigma^{q}\right)$ are topologically mixing. If $\Sigma_{M_{i}}$ is a periodic orbit, the desired result is trivial.

\section{The maximal measure}

In this section we consider a fixed $\Omega_{i}$ satisfying $h_{\text {top }}\left(\Omega_{i}\right)>0$, i.e. $\Omega_{i}$ is not only a periodic orbit. Therefore we denote $\Omega_{i}, F_{i}, M_{i}, D_{i}$ simply by $\Omega, F, M, D$ respectively. $\Omega \subset \Sigma_{f}^{+} \subset \Sigma_{n}^{+}$is expansive, hence $\Omega$ has at least one measure with maximal entropy (cf. [2]). It is proved in [3] that $(\Omega, \sigma)$ and $\left(\Sigma_{M}, \sigma\right)$ have isomorphic sets of maximal measures via $\mu \rightarrow \mu{ }^{\circ} \chi^{-1}$ and that there is a unique maximal measure $\mu$ on $\Sigma_{M}$ given by $\mu\left(0\left[y_{0} \cdots y_{k-1}\right]\right)=\pi_{y 0} P_{y_{0} y_{1}} \cdots P_{y_{k-2} y_{k-1}}$, where $\pi_{d}=u_{d} v_{d}, P_{d e}=M_{d e} v_{e} / \lambda v_{d}$ $(d, e \in D) . u$ is the unique (up to constant factors) positive left and $v$ the unique positive right eigenvector of $M$ for the eigenvalue $\lambda$ satisfying $\Sigma u_{d} v_{d}=1 . \lambda=r(M)$ is the spectral radius of the $l^{1}$-operator $w \rightarrow w M\left(w \in l^{1}\right) . \lambda>1$, because $\log \lambda=$ $h_{\text {top }}(\Omega)>0 . \S 3$ of $[3]$ shows that $h_{\text {top }}(\Omega) \leq \log \lambda$. The converse inequality follows from the variational principle (cf. [2]): $h_{\text {top }}(\Omega) \geq h(\mu)=\log \lambda$.

$\pi$ is a vector and $P$ is a matrix with index set $D$ satisfying $\pi_{d}>0, P_{d e} \geq 0(d, e \in D)$, $\pi P=\pi, \sum_{e \in D} P_{d e}=1(d \in D)$ and $\sum_{d \in D} \pi_{d}=1$. Hence $\pi$ and $P$ give rise to a Markov chain with countable state space. Assuming that $M$ and hence also $P$ are aperiodic, we can use the results proved in probability theory (cf. [1]).

$$
\begin{gathered}
(\pi, P) \text { is recurrent. } \\
P_{d e}^{(n)} \rightarrow \pi_{e}(n \rightarrow \infty) \text { for } d, e \in D .
\end{gathered}
$$

$P_{d e}^{(n)}$ denotes an entry of the matrix $P^{n}$. It follows from (3.2) that

$\mu\left({ }_{0}\left[y_{0} \cdots y_{i-1}\right] \cap_{k}\left[z_{0} \cdots z_{j-1}\right]\right) \quad$ converges to $\quad \mu\left({ }_{0}\left[y_{0} \cdots y_{i-1}\right]\right) \mu\left({ }_{0}\left[z_{0} \cdots z_{j-1}\right]\right)$ as $k \rightarrow \infty$.

Hence $\mu$ is mixing. This also completes the proof of theorem $2 . M_{d e}^{(n)}$ is the number of admissible blocks of length $n+1$ in $\Sigma_{M}$ beginning with $d$ and ending with $e$. Therefore $p_{n}=\sum_{d \in D} M_{d d}^{(n)}$ is the number of periodic points of period $n$ in $\Sigma_{M}$ ( $p_{n}<\infty$ by remark (i) after lemma 4). Set $\mu_{n}=\left(1 / p_{n}\right) \sum_{y \in Q_{n}} \delta_{y}$, where $Q_{n}$ is the set of all periodic points of period $n$ in $\Sigma_{M}$ and $\delta_{y}$ the measure concentrated in $y \in \Sigma_{M}$.

THEOREM 3. If $M$ is aperiodic, we have:

(i) $\lim _{n \rightarrow \infty} \lambda^{-n} p_{n}=1$.

(ii) $\mu_{n}$ converges in the weak topology to the unique maximal measure $\mu$ of $\Sigma_{M}$.

Proof. Choose a sequence $n_{i}$ of integers such that $\lambda^{-n_{i}} p_{n_{i}}$ converges to $C(0 \leq C \leq \infty)$. The sequence $\mu_{n_{i}}$ has a limit point $\nu$. We suppose that it converges (take again a 
subsequence, if necessary). We have

$$
\begin{aligned}
\mu_{n_{i}}\left(0\left[y_{0} \cdots y_{k-1}\right]\right) & =p_{n_{i}}^{-1} \operatorname{card}\left\{\mathbf{x} \in Q_{n_{i}}: x_{m}=y_{m} \quad \text { for } 0 \leq m \leq k-1\right\} \\
& =p_{n_{i}}^{-1} M_{y_{k}-1 y_{0}}^{\left(n_{i}-k+1\right)} \\
& =p_{n_{i}}^{-1} \lambda^{n_{i}} \lambda^{-k+1} P_{y_{k-1} y_{0}}^{\left(n_{i}-k+1\right)} v_{y_{k-1}} / v_{y_{0}} \\
& \rightarrow C^{-1} \lambda^{-k+1} \pi_{y_{0}} v_{y_{k-1}} / v_{y_{0}} \\
& =C^{-1} \pi_{y_{0}} P_{y_{0} y_{1}} \cdots P_{y_{k-2} y_{k-1}} \\
& =C^{-1} \mu\left({ }_{0}\left[y_{0} \cdots y_{k-1}\right]\right) .
\end{aligned}
$$

Hence $\nu\left({ }_{0}\left[y_{0} \cdots y_{k-1}\right]\right)=C^{-1} \mu\left({ }_{0}\left[y_{0} \cdots y_{k-1}\right]\right)$. As $\nu$ is a probability measure, we have $C=1$ proving (i). But then the computation above shows that every limit point of $\mu_{n}$ is $\mu$. This is (ii).

If $M$ has period $q>1$, then $\Omega=X_{1} \cup \cdots \cup X_{q}$ and every $X_{j}$ is an $\Omega_{i}$ for the piecewise monotonic transformation $f^{q}$. In the proof of theorem 2 we have computed $\tilde{M}$ corresponding to $\left(X_{i}, \sigma^{q}\right)$, which has period 1. Applying theorem 3 to $\tilde{M}$ we have:

COROLlARY. If $M$ has period $q$, we have:

(i) $p_{n}=0$, if $q$ is not a divisor of $n, \lim _{n \rightarrow \infty} p_{n q} \lambda^{-n q}=1$.

(ii) $\mu_{n q}$ converges in the weak topology to the unique maximal measure $\mu$ of $\Sigma_{M}$.

By lemmas 4, 5 and the second assertion of lemma 6 these results are also valid for $\Omega$.

Now we turn to a result about the number $b_{k}$ of admissible blocks of length $k$ in $\Omega$. For this we need that the left eigenvector $u$ of $M$ is in $l^{1}$ (the proof for this result is not published).

THEOREM 4. If $M$ is aperiodic, we have that $\lim _{k \rightarrow \infty} \lambda^{-k} b_{k}$ exists and is greater than zero.

Proof. Without loss of generality assume that $F=F_{i}=\Sigma_{f}^{+}$, considering $f \mid \phi^{-1}\left(F_{i}\right)$ instead of $f$. Set $D^{\prime}=\{0[k]: 1 \leq k \leq n\} \subset D$. $b_{k}$ is the number of admissible blocks of length $k$ in $\Sigma_{M}$ which begin with an element of $D^{\prime}$, because of the 1-1 correspondence of special paths in $M$ and points in $\Sigma_{f}^{+}$. Hence

$$
b_{k}=\sum_{d \in D^{\prime}} \sum_{e \in D} M_{d e}^{(k-1)}
$$

We have for $d \in D^{\prime}$ and $e \in D$

$$
\begin{aligned}
\lambda^{-m} M_{d e}^{(m)} & =P_{d e}^{(m)} v_{d} v_{e}^{-1}=\pi_{d}^{-1} v_{d} v_{e}^{-1} \pi_{d} P_{d e}^{(m)} \\
& \leq\left(u_{d} v_{e}\right)^{-1} \sum_{g \in D} \pi_{g} P_{g e}^{(m)}=\left(u_{d} v_{e}\right)^{-1} \pi_{e} \\
& =u_{e} / u_{d} \leq c^{-1} u_{e}, \quad \text { where } c=\min _{d \in D^{\prime}} u_{d}>0
\end{aligned}
$$

As $u \in l^{1}$, we have for every $\varepsilon>0$ a subset $D_{\varepsilon}$ of $D$ with $D \backslash D_{\varepsilon}$ finite, such that

$$
\sum_{e \in D_{e}} \lambda^{-m} M_{d e}^{(m)}<\varepsilon \quad \text { for all } m \geq 1
$$


Hence we can interchange the limit and the sums.

$$
\begin{aligned}
\lim _{k \rightarrow \infty} \lambda^{-k} b_{k} & =\lambda^{-1} \sum_{d \in D^{\prime}} \sum_{e \in D} \lim P_{d e}^{(k-1)} v_{d} / v_{e} \\
& =\lambda^{-1} \sum_{d \in D^{\prime}} \sum_{e \in D} \pi_{e} v_{d} / v_{e}=\lambda^{-1} \sum_{d \in D^{\prime}} \sum_{e \in D} u_{e} v_{d} .
\end{aligned}
$$

This is a positive constant, since $u \in l^{1}$.

As for theorem 3 we can generalize this result to the periodic case.

\section{Absolutely continuous invariant measures}

We consider the problem of finding an invariant measure $\mu$ on $([0,1], f)$, which is absolutely continuous with respect to the Lebesgue measure $\lambda$ on $[0,1]$. It suffices to consider this problem for $\phi^{-1}\left(\Omega_{i}\right)$ and $\phi^{-1}\left(\tilde{\Omega}_{i}\right)$ (we assume that $\phi$ is injective and denote $\phi^{-1}\left(\Omega_{i}\right)$ again by $\left.\Omega_{i}\right)$, because every invariant measure is a linear combination of measures concentrated on these sets.

If $f$ is piecewise $C^{2}$ and $\left|f^{\prime}(x)\right| \geq d>1$ for all $x \in[0,1]$, the sets $L_{j}$ considered in [6] are exactly those $\Omega_{i}$, which are finite unions of intervals. There is exactly one ergodic invariant measure $\mu$ absolutely continuous with respect to $\lambda$ on every $L_{j}$. All other $\Omega_{S}$ and all $\tilde{\Omega}_{S}$ have Lebesgue measure zero (theorem 2 of [6]).

In [5] one finds an example of an $f$ on $[0,1]$, piecewise $C^{2}, f^{\prime}(x)>1$ for $x \in(0,1]$ and $f^{\prime}(0)=1$, which has no finite invariant measure absolutely continuous with respect to $\lambda$. Below, we give an example of an $f$, piecewise $C^{1}, f^{\prime}(x) \geq 2$ for all $x$, which has a Cantor set $\Omega_{1}$ with $\lambda\left(\Omega_{1}\right)>0$ and $\lambda / \Omega_{1}$ is $f$-invariant.

We consider $[0,2]$ instead of $[0,1]$. Define $f$ on $(1,2]$ by $f(x)=2 x-1$ for $1<x \leq \frac{3}{2}$ and $2 x-2$ for $\frac{3}{2}<x \leq 2$ (or in any other way such that $\left.f((1,2]) \subset(1,2]\right) . \Omega_{2}=[1,2]$.

Now consider $[0,1]$. Set $a_{i}=c / i^{2} 2^{i-1}$, for some $c$ with $0<c<\left(\sum_{i=1}^{\infty} i^{-2}\right)^{-1}$. We define open sub-intervals $A_{m}^{k}\left(1 \leq k \leq 2^{m-1}, m \geq 1\right)$ of $[0,1] . A_{1}^{1}$ has midpoint $\frac{1}{2}$ and length $a_{1}$. Let $B_{2}^{1}$ and $B_{2}^{2}$ be the two closed sub-intervals of which $[0,1] \backslash A_{1}^{1}$ consists. The midpoint of $A_{2}^{k}$ is the midpoint of $B_{2}^{k}$ and the length of $A_{2}^{k}$ is $a_{2}(k=1,2)$. The $m$ th step is as follows. Let $B_{m}^{k}\left(1 \leq k \leq 2^{m-1}\right)$ be the closed intervals of equal length of which

$$
[0,1] \backslash \bigcup_{i=1}^{m-1} \bigcup_{j=1}^{2 i-1} A_{i}^{j}
$$

consists. Then the midpoint of $A_{m}^{k}$ is the midpoint of $B_{m}^{k}$ and the length of $A_{m}^{k}$ is $a_{m}\left(1 \leq k \leq 2^{m-1}\right)$. The $A_{m}^{k} \mathrm{~s}$ are pairwise disjoint, because the length of all the $A_{m}^{k} \mathrm{~s}$ $\left(1 \leq k \leq 2^{m-1}, m \geq 1\right)$ together is

$$
\sum_{m=1}^{\infty} 2^{m-1} a_{m}=\sum_{m=1}^{\infty} c m^{-2}<1 .
$$

Set

$$
C=[0,1] \backslash \bigcup_{i=1}^{\infty} \bigcup_{i=1}^{2 i-1} A_{i}^{j}=\bigcap_{i=2}^{\infty} \bigcup_{j=1}^{2 i-1} B_{i}^{i}
$$


To define $f$ on $[0,1]$, we define first $f^{\prime}=g \in C([0,1])$. Set $g(x)=2$ for $x \in C$. On $A_{m}^{k}$ define $g$ such that it is greater than or equal to 2 and continuous (i.e. $\lim g(x)=2$, where $x \in A_{m}^{k}$ approaches one of the endpoints of $A_{m}^{k}$ and

$$
\left.\sup \left\{|g(x)-2|: x \in \bigcup_{k} A_{m}^{k}\right\} \rightarrow 0 \quad \text { as } m \rightarrow \infty\right)
$$

and that

$$
\int_{A_{m}^{k}} g(x) d x= \begin{cases}a_{m-1} & \text { for } m \geq 2 \\ 1 & \text { for } m=1\end{cases}
$$

This is possible, because $a_{i} / a_{i+1}=2[(i+1) / i]^{2} \downarrow 2$, if $i \rightarrow \infty$. Define

$$
f(x)= \begin{cases}\int_{0}^{x} g(t) d t & \text { for } x \in B_{1}^{2} \cup A_{1}^{1} \\ \int_{d}^{x} g(t) d t & \text { for } x \in B_{2}^{2}\end{cases}
$$

where $d=\frac{1}{2}\left(1+a_{1}\right)$ is the initial point of $B_{2}^{2}$. Then $f / B_{1}^{2} \cup A_{1}^{1}$ and $f / B_{2}^{2}$ are increasing and $C^{1} \cdot f^{\prime}(x)=g(x) \geq 2$ for $x \in[0,1] \backslash\{d\}$. We have

$$
\lambda\left(B_{m}^{k} \cap C\right)=2^{-m+1}\left(1-\sum_{i=1}^{m-1} 2^{i-1} a_{i}\right)-\sum_{i=1}^{\infty} 2^{i-1} a_{i+m-1} .
$$

From this it follows that

$$
2 \lambda\left(B_{m}^{k} \cap C\right)=\lambda\left(B_{m-1}^{k^{\prime}} \cap C\right),
$$

where $k^{\prime}=k\left(\bmod 2^{m-2}\right)$. We show that $f\left(A_{m}^{k}\right)=A_{m-1}^{k^{\prime}}(=[1,2]$, if $m=1)$. To this end we prove that $\lambda\left(f\left(A_{m}^{k}\right)\right)=\lambda\left(A_{m-1}^{k^{\prime}}\right)(=\lambda([1,2])$, if $m=1)$ and $\lambda\left(f\left(B_{m}^{k}\right)\right)=$ $\lambda\left(B_{m-1}^{k^{\prime}}\right)$ (set $\left.B_{1}^{1}=[0,1]\right)$. The first assertion follows because of (4.2). For the second assertion remark that

$$
B_{m}^{k}=\left(C \cap B_{m}^{k}\right) \cup A_{m}^{k} \cup A_{m+1}^{2 k-1} \cup A_{m+1}^{2 k} \cdots .
$$

This is a union of disjoint sets. Hence

$$
\begin{aligned}
\lambda\left(f\left(B_{m}^{k}\right)\right) & =\int_{B_{m}^{k}} g(x) d x=\int_{C \cap B_{m}^{k}} 2 d x+\int_{A_{m}^{k}} g(x) d x+\cdots \\
& =2 \lambda\left(B_{m}^{k} \cap C\right)+a_{m-1}+2 a_{m}+4 a_{m+1}+\cdots \\
& =\lambda\left(B_{m-1}^{k^{\prime}} \cap C\right)+\lambda\left(A_{m-1}^{k^{\prime}}\right)+\lambda\left(A_{m}^{2 k^{\prime}-1} \cup A_{m}^{2 k^{\prime}}\right)+\cdots \\
& =\lambda\left(B_{m-1}^{k^{\prime}}\right) .
\end{aligned}
$$

Because $f \mid B_{2}^{1} \cup A_{1}^{1}$ and $f \mid B_{2}^{2}$ are increasing and $[0,1]$ is the disjoint union of the intervals $A_{i}^{j}\left(1 \leq j \leq 2^{i-1}, 1 \leq i \leq m-1\right)$ and $B_{m}^{i}\left(1 \leq i \leq 2^{m-1}\right)$, it follows that $f\left(A_{m-1}^{k}\right)=A_{m-2}^{k^{\prime}}$ and $f\left(B_{m}^{k}\right)=B_{m-1}^{k^{\prime}}(m \geq 2)$.

Now it follows that

$$
\Omega_{1}=\bigcap_{k=1}^{\infty} f^{-k}([0,1])=[0,1] \backslash \bigcup_{i=1}^{\infty} \bigcup_{j=1}^{2 i-1} A_{i}^{i}=C .
$$

By (4.3) we have that $\lambda / \Omega_{1}$ is invariant and by (4.1) that $\lambda\left(\Omega_{1}\right)>0$. 


\section{REFERENCES}

[1] L. Breiman. Probability. Addison-Wesley: Reading, Mass, 1968.

[2] M. Denker, C. Grillenberger \& K. Sigmund. Ergodic Theory on Compact Spaces: Springer Lecture Notes in Math. no. 527. Springer: Berlin, 1976.

[3] F. Hofbauer. On intrinsic ergodicity of piecewise monotonic transformations with positive entropy. Israel J. Math. 34 (1979), 213-237.

[4] F. Hofbauer. On intrinsic ergodicity of piecewise monotonic transformations with positive entropy II. Israel J. Math. 38 (1981), 107-115.

[5] A. Lasota \& J. Yorke. On the existence of invariant measures for piecewise monotonic transformations. Trans. AMS 186 (1973), 481-488.

[6] T. Li \& J. Yorke. Ergodic transformations from an interval into itself. Trans. AMS 235 (1978), 183-192.

[7] L. S. Young. A closing lemma on the interval. Invent. Math. 54 (1979), 179-187. 